MATHEMATICS OF COMPUTATION

Volume 74, Number 252, Pages 1803-1829

S 0025-5718(05)01754-0

Article electronically published on March 14, 2005

\title{
LOCALLY SUPPORTED RATIONAL SPLINE WAVELETS ON A SPHERE
}

\author{
DANIELA ROŞCA
}

\begin{abstract}
In this paper we construct certain continuous piecewise rational wavelets on arbitrary spherical triangulations, giving explicit expressions of these wavelets. Our wavelets have small support, a fact which is very important in working with large amounts of data, since the algorithms for decomposition, compression and reconstruction deal with sparse matrices. We also give a quasi-interpolant associated to a given triangulation and study the approximation error. Some numerical examples are given to illustrate the efficiency of our wavelets.
\end{abstract}

\section{INTRODUCTION}

For many fields of numerical analysis, wavelet-based methods have become popular since they provide efficient and fast algorithms.

For applications in geodesy or meteorology, where the sphere is taken as a model of the Earth, wavelets on the two-dimensional sphere are needed. In these applications one represents functions which estimate temperature, pressure, rainfall, ozone, etc., over the sphere $\mathbb{S}^{2}$, based on a discrete sample of measurements.

Another application is the modelling of closed surfaces as the graph of a function defined on the sphere.

Although the sphere appears to be a simple manifold, techniques from $\mathbb{R}^{2}$ do not easily extend to the sphere.

It is possible to reduce the approximation of a function defined on $\mathbb{S}^{2}$ to the approximation of a function defined on $[0,1] \times[0,1]$, but when using this approach, some periodicity conditions should be satisfied. If, for example, we consider the mapping

$$
\rho: U=[0,1] \times[0,1] \rightarrow \mathbb{S}^{2},(\varphi, \theta) \stackrel{\rho}{\rightarrow}\left(\begin{array}{l}
\cos (2 \pi \varphi) \sin (\pi \theta) \\
\sin (2 \pi \varphi) \sin (\pi \theta) \\
\cos (\pi \theta)
\end{array}\right),
$$

then a function defined on $U$ can be identified with a function on $\mathbb{S}^{2}$. But not every continuous function on $U$ gives rise to a $C^{0}$ function on $\mathbb{S}^{2}$, since for example the lines $\{(\varphi, 0) \mid \varphi \in[0,1]\}$ and $\{(\varphi, 1) \mid \varphi \in[0,1]\}$ collapse into a point. To make sure that a continuous function $f$ defined on the rectangle $U$ remains continuous after

Received by the editor October 3, 2003 and, in revised form, April 12, 2004.

2000 Mathematics Subject Classification. Primary 42C40, 41A63; Secondary 41A15, 65D07, $41 \mathrm{~A} 17$.

Key words and phrases. Wavelets, multivariate approximation, interpolation.

Research supported by the EU Research Training Network MINGLE, HPRN-CT-1999-00117. 
mapping it onto $\mathbb{S}^{2}$, it is necessary that $f$ satisfy the following conditions:

$$
\left\{\begin{array}{l}
f(0, \theta)=f(1, \theta), 0 \leq \theta \leq 1, \\
\text { there exist constants } \mathcal{S}_{N}, \mathcal{S}_{S} \text { such that }\left\{\begin{array}{l}
f(\varphi, 0)=\mathcal{S}_{N}, \\
f(\varphi, 1)=\mathcal{S}_{S},
\end{array} \quad 0 \leq \varphi \leq 1 .\right.
\end{array}\right.
$$

Unfortunately, such conditions are not easily satisfied.

We will consider in this paper another approach, where we make use of a radial projection onto the sphere. Before summarizing the content of the present work, we review some approaches which treat the sphere $\mathbb{S}^{2}$.

The first construction of wavelets on the sphere has been presented by Dahlke et al. in [3] using a tensor product basis, where one factor is an exponential spline. The multiresolution is nonstationary and the wavelets are $C^{1}$, have global support and are semi-orthogonal. They also give a characterization of $C^{1}$ functions on $\mathbb{S}^{2}$ and $\mathbb{S}^{3}$ with a wavelet representation. Their construction is based on an approach which used splines, proposed by Schumaker and Traas in 23 .

Another approach to creating wavelets on the $2 \mathrm{D}$ sphere is the one realized by Potts and Tasche in [15]. They first map the rectangle $[0, \pi] \times[0,2 \pi]$ to the sphere via standard spherical coordinates and then construct nonorthogonal wavelets by taking the tensor product of interpolatory trigonometric wavelets and algebraic polynomial wavelets, obtaining continuous wavelets on $\mathbb{S}^{2}$. Doing this, singularities and distortions near the poles occur. A similar idea with spherical harmonics is presented in [16, where the authors construct a frame in $L^{2}\left(\mathbb{S}^{2}\right)$ consisting of smooth functions arising from kernels of spherical harmonics. The idea of constructing spherical wavelets using spherical harmonics was realized in a different manner in [8] for equidistant nodes and [12] for scattered data. A drawback is that the spherical harmonic functions are globally supported and suffer from the same difficulties as Fourier representations on the line, such as "ringing".

In 11, Narcowich and Ward construct a nonstationary multiresolution analysis with functions generated by translations of a spherical basis function. The wavelets here are orthogonal and localized, but not locally supported. In 24, Weinreich describes a nonstationary multiresolution and biorthogonal $C^{1}$ wavelets on the 2D sphere via tensor product. In [21, Schröder and Sweldens present a method to obtain biorthogonal wavelets on spherical triangulations using the lifting scheme. This approach is useful in practical areas (e.g. for compression of tomographic data) as well as in computer graphics, but no result regarding the stability was given. For the wavelets obtained by lifting, the stability was established later by Cohen et al. in 2. Here, finite element wavelets on planar triangulations with compactly supported duals are obtained by the lifting scheme.

In 10] Lounsbery et al. construct wavelets defined on subdivision surfaces. These surfaces are constructed by iteratively refining a control polyhedron $M^{0}$, so that the sequence of refined polyhedra $M^{1}, M^{2}, \ldots$ converges to the sphere. Taking the limit of this sequence of wavelets, they construct globally supported wavelets defined on the sphere which are then truncated to a small region. Thus, they produce functions that are no longer elements of the orthogonal complementary wavelet spaces and they call them quasi-wavelets.

Piecewise constant wavelets on arbitrary spherical triangulations were constructed by Nielson et al. in [13] and Bonneau in [1]. Their wavelets are "nearly orthogonal" and no Riesz stability was proved. The techniques presented in this paper also work for piecewise constant wavelets. Thus, in [17] we enlarged the classes of 
wavelets constructed by Bonneau and by Nielson et al., establishing at the same time the Riesz stability. In [18, we realized a comparison of the wavelets obtained in [17, with respect to the $l_{2}$-norm of the reconstruction error.

Our construction is based on the results of Floater and Quak for planar triangulations, presented in [4, 5] and [6]. In a first step we transfer their results to a no longer planar triangulation having its vertices in 3 -space. Then, using a radial projection, we obtain locally supported wavelets defined on the sphere. Explicit and simple expressions are available.

The present work is structured as follows.

In Section 2 we prove some statements that are necessary to use the results obtained in [4, [5] and [6] and we introduce a norm on $\mathbb{S}^{2}$ which is equivalent to the usual $L_{2}$-norm of $\mathbb{S}^{2}$.

In Section 3 the construction of a stationary multiresolution analysis on the sphere $\mathbb{S}^{2}$ will be described and explicit formulas for the scaling functions will be given.

By definition, a multiresolution analysis of the space $L^{2}\left(\mathbb{S}^{2}\right)$ is a sequence of subspaces $\left\{\mathcal{V}^{j}: j \geq 0\right\}$ of $L^{2}\left(\mathbb{S}^{2}\right)$ which satisfy the following requirements:

$$
\begin{aligned}
& \mathcal{V}^{j} \subseteq \mathcal{V}^{j+1} \text { for all } j \in \mathbb{N}_{0} . \\
& \operatorname{clos}_{L^{2}\left(\mathbb{S}^{2}\right)} \bigcup_{j=0}^{\infty} \mathcal{V}^{j}=L^{2}\left(\mathbb{S}^{2}\right) .
\end{aligned}
$$

(3) There are index sets $\mathcal{K}_{j} \subseteq \mathcal{K}_{j+1}$ such that for every level $j$ there exists a Riesz basis $\left\{\varphi_{v}^{j}, v \in \mathcal{K}_{j}\right\}$ of the space $\mathcal{V}^{j}$. This means that there exist constants $0<c \leq C<\infty$, independent of the level $j$, such that

$$
c 2^{-j}\left\|\left\{c_{v}^{j}\right\}_{v \in \mathcal{K}^{j}}\right\|_{l_{2}\left(\mathcal{K}_{j}\right)} \leq\left\|\sum_{v \in \mathcal{K}^{j}} c_{v}^{j} \varphi_{v}^{j}\right\|_{L^{2}\left(\mathbb{S}^{2}\right)} \leq C 2^{-j}\left\|\left\{c_{v}^{j}\right\}_{v \in \mathcal{K}^{j}}\right\|_{l_{2}\left(\mathcal{K}_{j}\right)} .
$$

We do not require the scaling functions $\varphi_{v}^{j}$ to be translations and dilations of the same function $\varphi$. In some papers the authors replace the translation requirement with a rotational one, but in most of the research on spherical wavelets this requirement is not demanded since it is difficult to be satisfied.

Once the multiresolution analysis is determined, we construct the wavelet spaces $\mathcal{W}^{j}$. They are orthogonal complements with respect to a weighted $L^{2}$-inner product. The basis functions of each space $\mathcal{W}^{j}$ are commonly called wavelets. In Section 4 we describe the construction of a locally supported wavelet basis of $\mathcal{W}^{j}$. Our wavelets will be semi-orthogonal, meaning that we have orthogonality between levels but not within one level. Some authors use the term prewavelets instead of semi-orthogonal wavelets. Then, in Section 5 we present a quasi-interpolant and prove some error estimates for the approximation. Finally, in Section [6 we give some numerical examples.

The construction presented in this paper may also be adapted for arbitrary sphere-like surfaces. A sphere-like surface is defined as $\left\{\sigma(v)=\rho(v) v, v \in \mathbb{S}^{2}\right\}$, with a continuous positive function $\rho$ defined on $\mathbb{S}^{2}$. We restrict our attention to the case of the $2 \mathrm{D}$ sphere $\mathbb{S}^{2}$, since this case has more practical applications and since we can obtain an explicit expression for our wavelets defined on $\mathbb{S}^{2}$. The conditions that have to be satisfied by the function $\rho$ in order to assure the Riesz stability of the wavelets are given in [19]. 


\section{BASICS}

2.1. Spatial triangulations with planar triangles. Consider the unit sphere $\mathbb{S}^{2}$ of $\mathbb{R}^{3}$ with the center in $O$, and let $\Pi$ be a convex polyhedron having all the vertices situated on $\mathbb{S}^{2}$ and triangular faces 1 such that no face contains $O$ and $O$ is situated inside the polyhedron. We denote by $\mathcal{T}=\left\{T_{1}, \ldots, T_{M}\right\}$ the set of the faces of $\Pi$, by $E$ the set of edges and by $V$ the set of vertices. For a vertex $v \in V$, the set of neighbors of $v$ is $V_{v}=\{w \in V:[v, w] \in E\}$. The surface of the polyhedron $\Pi$ will be denoted by $\Omega$. Given the data values $f_{v} \in \mathbb{R}$, for $v \in V$, there is a unique function $f: \Omega \rightarrow \mathbb{R}$, which is continuous on $\Omega$, linear on each triangle in $\mathcal{T}$, and which interpolates the data: $f(v)=f_{v}, v \in V$. For a given $\Pi$, the set of all such continuous and piecewise linear functions forms a linear space $S$ with dimension $|V|$. A basis for $S$ is $\left\{\phi_{v}, v \in V\right\}$, where $\phi_{v}: \Omega \rightarrow \mathbb{R}$ is the unique continuous and piecewise linear "hat" function in $S$ such that for all $w \in V$,

$$
\phi_{v}(w)= \begin{cases}1 & \text { for } w=v, \\ 0 & \text { otherwise. }\end{cases}
$$

Specifically, the "hat" function $\phi_{M_{1}}: \Omega \rightarrow \mathbb{R}$, associated to the vertex $M_{1}\left(x_{1}, y_{1}, z_{1}\right)$ $\in \Omega$ is given by

$$
\phi_{M_{1}}(x, y, z)=\left\{\begin{array}{ccc}
\left|\begin{array}{ccc}
x & y & z \\
x_{i} & y_{i} & z_{i} \\
x_{k} & y_{k} & z_{k}
\end{array}\right| & \\
\left|\begin{array}{ccc}
x_{1} & y_{1} & z_{1} \\
x_{i} & y_{i} & z_{i} \\
x_{k} & y_{k} & z_{k}
\end{array}\right| & \text { on each triangle }\left[M_{1} M_{i} M_{k}\right] \text { of } \mathcal{T}, \\
& 0 &
\end{array}\right.
$$

We intend to use some of the results from [4], [5] and [6]. For this, we need to prove the following lemmas.

The first lemma gives a formula for the integral of the product of two linear functions defined on a triangle $T$ of $\Omega$. The proof of this lemma is given in the Appendix.

Lemma 1. Let $T=\left[M_{1} M_{2} M_{3}\right] \subset \Omega$ with $M_{i}\left(x_{i}, y_{i}, z_{i}\right), \phi_{1}(x, y, z)=a x+b y+c z$, $\phi_{2}(x, y, z)=m x+n y+p z$ and denote $f_{i}=\phi_{1}\left(x_{i}, y_{i}, z_{i}\right), g_{i}=\phi_{2}\left(x_{i}, y_{i}, z_{i}\right), i=$ $1,2,3$. Then

$$
\int_{T} \phi_{1}(\mathbf{x}) \phi_{2}(\mathbf{x}) d \Omega(\mathbf{x})=\frac{a(T)}{12}\left(f_{1} g_{1}+f_{2} g_{2}+f_{3} g_{3}+\left(f_{1}+f_{2}+f_{3}\right)\left(g_{1}+g_{2}+g_{3}\right)\right) \text {. }
$$

Let $\langle\cdot, \cdot\rangle_{\Omega}$ be the following inner product, based on the given polyhedron

$$
\langle f, g\rangle_{\Omega}=\sum_{T \in \mathcal{T}} \frac{1}{a(T)} \int_{T} f(\mathbf{x}) g(\mathbf{x}) d \mathbf{x}, f, g \in C(\Omega),
$$

where $a(T)$ is the area of the triangle $T$. We also consider the induced norm $\|f\|_{\Omega}=\langle f, f\rangle_{\Omega}^{1 / 2}$.

Regarding this norm, we prove the following equivalence.

\footnotetext{
${ }^{1}$ The polyhedron could also have faces which are not triangles. In this case we triangulate each of these faces and consider it as having triangular faces, with some of the faces coplanar triangles.
} 
Lemma 2. In the space $L^{2}(\Omega)$, the norm $\|\cdot\|_{\Omega}$ is equivalent to the usual norm $\|\cdot\|_{L^{2}(\Omega)}$.

Proof. For $f \in L^{2}(\Omega)$,

$$
\begin{aligned}
\|f\|_{L^{2}(\Omega)}^{2} & =\int_{\Omega} f^{2}(\mathbf{x}) d \Omega(\mathbf{x}) \\
& =\sum_{T \in \mathcal{T}} \int_{T} f^{2}(\mathbf{x}) d \Omega(\mathbf{x}) .
\end{aligned}
$$

Now, using the definition of the norm $\|\cdot\|_{\Omega}$, we obtain

$$
\begin{aligned}
& \frac{1}{\max _{T \in \mathcal{T}} a(T)} \sum_{T \in \mathcal{T}} \int_{T} f^{2}(\mathbf{x}) d \Omega(\mathbf{x}) \\
& \leq \sum_{T \in \mathcal{T}^{0}} \frac{1}{a(T)} \int_{T} f^{2}(\mathbf{x}) d \Omega(\mathbf{x}) \\
& \leq \frac{1}{\min _{T \in \mathcal{T}} a(T)} \sum_{T \in \mathcal{T}} \int_{T} f^{2}(\mathbf{x}) d \Omega(\mathbf{x}),
\end{aligned}
$$

whence

$$
\frac{1}{\max _{T \in \mathcal{T}} a(T)}\|f\|_{L^{2}(\Omega)}^{2} \leq\|f\|_{\Omega}^{2} \leq \frac{1}{\min _{T \in \mathcal{T}} a(T)}\|f\|_{L^{2}(\Omega)}^{2}
$$

2.2. Spherical triangulations. Now for the given polyhedron $\Pi$, we define the radial projection onto $\mathbb{S}^{2}, p: \Omega \rightarrow \mathbb{S}^{2}$,

$$
p(x, y, z)=\left(\frac{x}{\sqrt{x^{2}+y^{2}+z^{2}}}, \frac{y}{\sqrt{x^{2}+y^{2}+z^{2}}}, \frac{z}{\sqrt{x^{2}+y^{2}+z^{2}}}\right), \quad(x, y, z) \in \Omega,
$$

and its inverse $p^{-1}: \mathbb{S}^{2} \rightarrow \Omega$

$$
p^{-1}\left(\eta_{1}, \eta_{2}, \eta_{3}\right)=\left(-\frac{\eta_{1} d}{a \eta_{1}+b \eta_{2}+c \eta_{3}},-\frac{\eta_{2} d}{a \eta_{1}+b \eta_{2}+c \eta_{3}},-\frac{\eta_{3} d}{a \eta_{1}+b \eta_{2}+c \eta_{3}}\right)
$$

where $a x+b y+c z+d=0$ is the equation of that face of $\Pi$ onto which the point $\left(\eta_{1}, \eta_{2}, \eta_{3}\right) \in \mathbb{S}^{2}$ projects. In the case when the point $\left(\eta_{1}, \eta_{2}, \eta_{3}\right)$ projects onto an edge, then we may choose one of its adjacent faces to express the function $p^{-1}$.

If we consider the images $U_{i}=p\left(T_{i}\right)$ of the triangles $T_{i}$ under the projection $p$, then we say that $\mathcal{U}=\left\{U_{1}, \ldots, U_{M}\right\}$ is a triangulation of the sphere $\mathbb{S}^{2}$. The functions $\varphi_{v}: \mathbb{S}^{2} \rightarrow \mathbb{R}, \varphi_{v}=\phi_{v} \circ p^{-1}, v \in V$ are continuous on $\mathbb{S}^{2}$ and their supports are $\mathcal{M}_{v}$ - the set of all spherical triangles of $\mathcal{U}$ that contain the vertex $v$, so the $\varphi_{v}$ are local around the vertex $v$. 
Remark 3. Let $\left[M_{1} M_{i} M_{k}\right]$ be a triangle of $\mathcal{T}$ and $\left[M_{1}^{\prime} M_{i}^{\prime} M_{k}^{\prime}\right]$ its radial projection onto $\mathbb{S}^{2}$. Then, the restriction to $\left[M_{1}^{\prime} M_{i}^{\prime} M_{k}^{\prime}\right]$ of $\varphi_{M_{1}}$ is

$$
\begin{aligned}
\varphi_{M_{1}}\left(\eta_{1}, \eta_{2}, \eta_{3}\right) & \\
& =\left|\begin{array}{ccc}
-\frac{\eta_{1} d}{a \eta_{1}+b \eta_{2}+c \eta_{3}} & -\frac{\eta_{2} d}{a \eta_{1}+b \eta_{2}+c \eta_{3}} & -\frac{\eta_{3} d}{a \eta_{1}+b \eta_{2}+c \eta_{3}} \\
x_{k} & y_{i} & z_{i} \\
y_{k} & z_{k}
\end{array}\right| \cdot\left|\begin{array}{ccc}
x_{1} & y_{1} & z_{1} \\
x_{i} & y_{i} & z_{i} \\
x_{k} & y_{k} & z_{k}
\end{array}\right|^{-1} \\
& =-\frac{d}{a \eta_{1}+b \eta_{2}+c \eta_{3}} \cdot\left|\begin{array}{ccc}
\eta_{1} & \eta_{2} & \eta_{3} \\
x_{i} & y_{i} & z_{i} \\
x_{k} & y_{k} & z_{k}
\end{array}\right| \cdot\left|\begin{array}{ccc}
x_{1} & y_{1} & z_{1} \\
x_{i} & y_{i} & z_{i} \\
x_{k} & y_{k} & z_{k}
\end{array}\right|-1
\end{aligned}
$$

where $a, b, c, d$ are the coefficients of $x, y, z, 1$ of the polynomial function

$$
\left|\begin{array}{cccc}
x & y & z & 1 \\
x_{1} & y_{1} & z_{1} & 1 \\
x_{i} & y_{i} & z_{i} & 1 \\
x_{k} & y_{k} & z_{k} & 1
\end{array}\right| .
$$

Thus the "hat" functions $\varphi_{v}$ are piecewise rational functions, with the numerator and denominator linear polynomials of degree one.

The following proposition establishes the relations between the area elements of $\mathbb{S}^{2}$ and $\Omega$.

Proposition 4. The relations between $d \Omega(\mathbf{x})$ (the area element of $\Omega$ ) and $d \omega(\boldsymbol{\eta})$ (the area element of $\mathbb{S}^{2}$ ) are

$$
\begin{aligned}
& d \omega(\boldsymbol{\eta})=\frac{|d|}{\sqrt{a^{2}+b^{2}+c^{2}}} \cdot \frac{1}{\left(x^{2}+y^{2}+z^{2}\right)^{3 / 2}} d \Omega(\mathbf{x}), \\
& d \Omega(\mathbf{x})=\frac{d^{2} \sqrt{a^{2}+b^{2}+c^{2}}}{\left|a \eta_{1}+b \eta_{2}+c \eta_{3}\right|^{3}} d \omega(\boldsymbol{\eta}),
\end{aligned}
$$

where $\mathbf{x}=(x, y, z) \in \Omega, \boldsymbol{\eta}=\left(\eta_{1}, \eta_{2}, \eta_{3}\right) \in \mathbb{S}^{2}$, and $a, b, c, d$ are determined for each face of $\Pi$ as described above.

The proof of this proposition can be found in the Appendix.

For $L^{2}$-integrable functions defined on $\mathbb{S}^{2}$, let $\langle\cdot, \cdot\rangle_{*}$ be defined by

$$
\langle F, G\rangle_{*}=\langle F \circ p, G \circ p\rangle_{\Omega} .
$$


Then $\langle\cdot, \cdot\rangle_{*}$ is an inner product which induces the norm $\|F\|_{*}=\langle F, F\rangle_{*}^{1 / 2}$ and we have

$$
\begin{aligned}
\langle F, G\rangle_{*} & =\sum_{T \in \mathcal{T}} \frac{1}{a(T)} \int_{T} F(p(\mathbf{x})) G(p(\mathbf{x})) d \Omega(\mathbf{x}) \\
& =\sum_{T \in \mathcal{T}} \frac{1}{a(T)} \int_{p(T)} F(\boldsymbol{\eta}) G(\boldsymbol{\eta}) \frac{d_{T}^{2} \sqrt{a_{T}^{2}+b_{T}^{2}+c_{T}^{2}}}{\left|a_{T} \eta_{1}+b_{T} \eta_{2}+c_{T} \eta_{3}\right|^{3}} d \omega(\boldsymbol{\eta}) \\
& =\sum_{T \in \mathcal{T}} \int_{p(T)} F(\boldsymbol{\eta}) G(\boldsymbol{\eta}) \frac{2 d_{T}^{2}}{\left|a_{T} \eta_{1}+b_{T} \eta_{2}+c_{T} \eta_{3}\right|^{3}} d \omega(\boldsymbol{\eta}),
\end{aligned}
$$

where $a_{T}, b_{T}, c_{T}, d_{T}$ are the coefficients of $x, y, z$ and 1 in the polynomial function given by (4), and the notation is as in Remark 3 ,

The inner product $\langle\cdot, \cdot\rangle_{*}$ can be interpreted as a "multi-weighted" inner product, with the weights

$$
w_{T}\left(\eta_{1}, \eta_{2}, \eta_{3}\right)=2 d_{T}^{2}\left|a_{T} \eta_{1}+b_{T} \eta_{2}+c_{T} \eta_{3}\right|^{-3} .
$$

The following lemma establishes a norm equivalence in $L^{2}\left(\mathbb{S}^{2}\right)$.

Lemma 5. In the space $L^{2}\left(\mathbb{S}^{2}\right)$, the norm $\|\cdot\|_{*}$ is equivalent to the usual norm $\|\cdot\|_{2}$ of $L^{2}\left(\mathbb{S}^{2}\right)$.

Proof. For $F \in L^{2}\left(\mathbb{S}^{2}\right)$,

$$
\|F\|_{*}^{2}=\sum_{T \in \mathcal{T}} \int_{p(T)} F^{2}(\boldsymbol{\eta}) \frac{2 d_{T}^{2}}{\left|a_{T} \eta_{1}+b_{T} \eta_{2}+c_{T} \eta_{3}\right|^{3}} d \omega(\boldsymbol{\eta}) .
$$

Denoting by $\min (T)$ and $\max (T)$ the minimum and maximum values of the functions

$$
h_{T}(\boldsymbol{\eta})=\left|a_{T} \eta_{1}+b_{T} \eta_{2}+c_{T} \eta_{3}\right|^{-3}
$$

respectively, a simple calculation shows that $\min (T)=\frac{1}{(2 a(T))^{3}}$ and $\max (T)=$ $\frac{1}{\left|d_{T}\right|^{3}}$. Thus, we write

and then

$$
\min (T) \leq \frac{1}{\left|a_{T} \eta_{1}+b_{T} \eta_{2}+c_{T} \eta_{3}\right|^{3}} \leq \max (T)
$$

$$
2 \sum_{T \in \mathcal{T}} d_{T}^{2} \min (T) \int_{p(T)} F^{2}(\boldsymbol{\eta}) d \omega(\boldsymbol{\eta}) \leq\|F\|_{*}^{2} \leq 2 \sum_{T \in \mathcal{T}} d_{T}^{2} \max (T) \int_{p(T)} F^{2}(\boldsymbol{\eta}) d \omega(\boldsymbol{\eta}) .
$$

Denoting $M=2 \max _{T \in \mathcal{T}}\left\{d_{T}^{2} \max (T)\right\}$ and $m=2 \min _{T \in \mathcal{T}}\left\{d_{T}^{2} \min (T)\right\}$, we finally obtain

$$
m\|F\|_{L^{2}\left(\mathbb{S}^{2}\right)}^{2} \leq\|F\|_{*}^{2} \leq M\|F\|_{L^{2}\left(\mathbb{S}^{2}\right)}^{2} .
$$

\section{Multiresolution analysis}

Given $\Omega$, we can say that $\mathcal{T}=\mathcal{T}^{0}$ is a triangulation of $\Omega$, and next we wish to consider its uniform refinement $\mathcal{T}^{1}$. For a given triangle $\left[M_{1} M_{2} M_{3}\right]$ in $\mathcal{T}^{0}$, let $A_{1}, A_{2}, A_{3}$ denote the midpoints of the edges $M_{2} M_{3}, M_{3} M_{1}$ and $M_{1} M_{2}$, respectively. Then we consider the set

$$
\mathcal{T}^{1}=\bigcup_{\left[M_{1} M_{2} M_{3}\right] \in \mathcal{T}^{0}}\left\{\left[M_{1} A_{2} A_{3}\right],\left[A_{1} M_{2} A_{3}\right],\left[A_{1} A_{2} M_{3}\right],\left[A_{1} A_{2} A_{3}\right]\right\},
$$


which is also a triangulation of $\Omega$, and continuing the refinement process in the same way, we obtain a triangulation $\mathcal{T}^{j}$ of $\Omega$ for $j \in \mathbb{N}$. We denote by $V^{j}$ the set of all vertices of the triangles in $\mathcal{T}^{j}$ and by $E^{j}$ the set of all edges of triangles in $\mathcal{T}^{j}$. Then $S^{j}, V_{v}^{j}, \phi_{v}^{j}, \varphi_{v}^{j}, \mathcal{U}^{j}$, and $\mathcal{M}_{v}^{j}$ are defined accordingly. The space $S^{j}$ is a subspace of $S^{j+1}$, since we have

$$
\phi_{v}^{j}=\phi_{v}^{j+1}+\frac{1}{2} \sum_{w \in V_{v}^{j+1}} \phi_{w}^{j+1}, v \in V^{j}, j \in \mathbb{N} .
$$

For the nodal functions $\left\{\phi_{v}^{j}\right\}$ the following statement holds.

Lemma 6. There exist constants $0<c \leq C<\infty$, independent of the level $j$, such that for all functions $g_{j}=\sum_{v \in V^{j}} c_{v}^{j} \phi_{v}^{j}$ we have

$$
c\left\|\left\{\bar{h}_{v}^{j} c_{v}^{j}\right\}_{v \in V^{j}}\right\|_{l_{2}} \leq\left\|g_{j}\right\|_{L^{2}(\Omega)} \leq C\left\|\left\{\bar{h}_{v}^{j} c_{v}^{j}\right\}_{v \in V^{j}}\right\|_{l_{2}} .
$$

Here $\bar{h}_{v}^{j}$ denotes the diameter of the support of $\phi_{v}^{j}$.

Proof. Due to the uniform refinement, it is clear that $\bar{h}_{v}^{j} \approx 2^{-j}$, so it is enough to prove the equivalence

$$
\left\|\left\{2^{-j} c_{v}^{j}\right\}_{v \in V^{j}}\right\|_{l_{2}} \approx\left\|\sum_{v \in V^{j}} c_{v}^{j} \phi_{v}^{j}\right\|_{L^{2}(\Omega)}
$$

or equivalently

$$
\left\|\left\{c_{v}^{j}\right\}_{v \in V^{j}}\right\|_{l_{2}} \approx\left\|\sum_{v \in V^{j}} c_{v}^{j} 2^{j} \phi_{v}^{j}\right\|_{L^{2}(\Omega)} .
$$

In order to prove this, we refer to Lemma 5.2 in 7 . As in that lemma, we can show that for all $f$ in $S^{j}$ we have

$$
\frac{2^{-2 j}}{12} \sum_{w \in V^{j}} t(w) f^{2}(w) \leq\|f\|_{\Omega}^{2} \leq \frac{2^{-2 j}}{3} \sum_{w \in V^{j}} t(w) f^{2}(w),
$$

where $t(w)$ is the number of the triangles in $\mathcal{T}^{j}$ that contain the vertex $w$.

If we take $f=\sum_{v \in V^{j}} c_{v}^{j} \phi_{v}^{j}$, then $f(w)=c_{w}^{j}$ since $\phi_{v}(w)=\delta_{v w}$. So,

$$
\frac{2^{-2 j}}{12} \sum_{w \in V^{j}} t(w)\left(c_{w}^{j}\right)^{2} \leq\left\|\sum_{w \in V^{j}} c_{w}^{j} \phi_{w}^{j}\right\|_{\Omega}^{2} \leq \frac{2^{-2 j}}{3} \sum_{w \in V^{j}} t(w)\left(c_{w}^{j}\right)^{2} .
$$

But $t(w)$ is either 6 or $t^{0}(w)$ from the initial triangulation, so if we denote $n=$ $\min _{v \in V^{0}}\left(6, t^{0}(v)\right)$ and $N=\max _{v \in V^{0}}\left(6, t^{0}(v)\right)$, then we may write

$$
\frac{n}{12} \sum_{w \in V^{j}}\left(c_{w}^{j}\right)^{2} \leq\left\|\sum_{w \in V^{j}} c_{w}^{j} 2^{j} \phi_{w}^{j}\right\|_{\Omega}^{2} \leq \frac{N}{3} \sum_{w \in V^{j}}\left(c_{w}^{j}\right)^{2},
$$

which together with Lemma 2 completes the proof.

The sequence of triangulations $\left\{\mathcal{T}^{j}\right\}_{j \in \mathbb{N}}$ is regular in the following sense: the ratios of the radii of the inscribed and circumscribed circles remain bounded from below by a constant $\gamma_{0}>0$ independent of the level $j$ and of the triangles. For each triangle $K_{j}$ of $\mathcal{T}^{j}$ the diameter $h\left(K_{j}\right)=\operatorname{diam} K_{j}$ characterizes its typical size. 


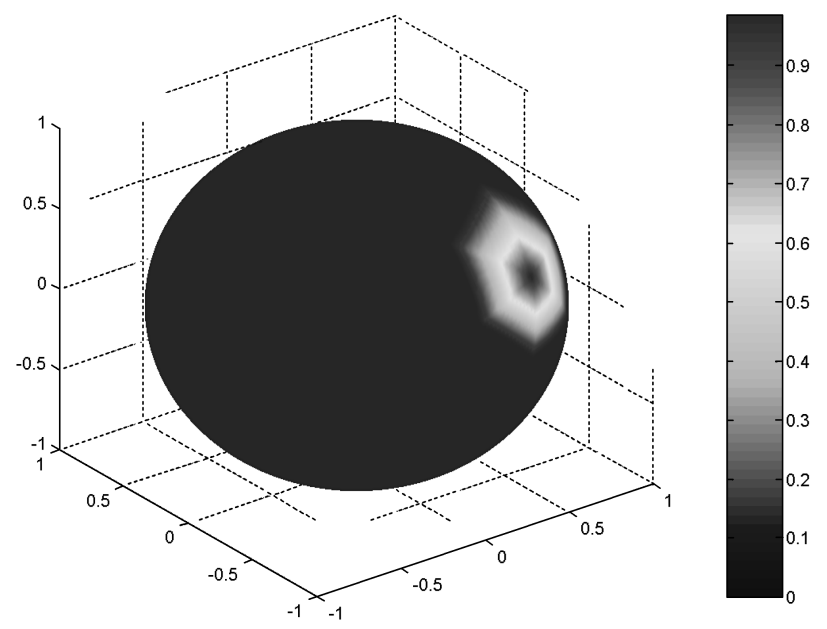

Figure 1. A pyramidal function $\varphi_{u}^{j}$ represented on $\mathbb{S}^{2}$.

Usually, $h\left(\mathcal{T}^{j}\right)=\max h\left(K_{j}\right)$ is called (maximal) mesh-size of $\mathcal{T}^{j}$. Let $h_{\min }\left(\mathcal{T}^{j}\right)=$ $\min h\left(K_{j}\right)$. The sequence of triangulations $\left\{\mathcal{T}^{j}\right\}_{j \in \mathbb{N}}$ is quasi-uniform, in the sense that the ratio $\frac{h\left(\mathcal{T}^{j}\right)}{h_{\min }\left(\mathcal{T}^{j}\right)}$ is bounded from above by a constant $\gamma_{1}<\infty$, independent of $j$.

If we set $\mathcal{V}^{j}=\operatorname{span}\left\{\varphi_{v}^{j}, v \in V^{j}\right\}, j \in \mathbb{N}$, then $\mathcal{V}^{j}$ is a subspace of $\mathcal{V}^{j+1}$, due to the refinement equation

$$
\varphi_{v}^{j}=\varphi_{v}^{j+1}+\frac{1}{2} \sum_{w \in V_{v}^{j+1}} \varphi_{w}^{j+1}, v \in V^{j}, j \in \mathbb{N} .
$$

The set $\left\{\varphi_{v}^{j}, v \in V^{j}\right\}$ is a local basis for $\mathcal{V}^{j}$ in the sense that $\operatorname{diam}\left(\operatorname{supp} \varphi_{v}^{j}\right) \approx 2^{-j}$.

Let $\left[M_{1} M_{i} M_{k}\right]$ be a triangle of a triangulation $\mathcal{T}^{j}$ and $\left[M_{1}^{\prime} M_{i}^{\prime} M_{k}^{\prime}\right]$ its radial projection onto $\mathbb{S}^{2}$. Then, using the same arguments as in Remark 3, we can deduce that the restriction to $\left[M_{1}^{\prime} M_{i}^{\prime} M_{k}^{\prime}\right]$ of $\varphi_{M_{1}}^{j}$ is

$$
\varphi_{M_{1}}^{j}\left(\eta_{1}, \eta_{2}, \eta_{3}\right)=\left|\begin{array}{ccc}
\eta_{1} & \eta_{2} & \eta_{3} \\
x_{i} & y_{i} & z_{i} \\
x_{k} & y_{k} & z_{k}
\end{array}\right| \cdot\left|\begin{array}{cccc}
\eta_{1} & \eta_{2} & \eta_{3} & 0 \\
x_{1} & y_{1} & z_{1} & 1 \\
x_{i} & y_{i} & z_{i} & 1 \\
x_{k} & y_{k} & z_{k} & 1
\end{array}\right|^{-1} .
$$

Thus the spaces $\mathcal{V}^{j}$ are spaces of rational splines, with the numerator and denominator linear polynomials of degree one. An example of function $\varphi_{u}^{j} \in \mathcal{V}^{j}$ is represented in Figure 1 .

\section{WAVELETS}

With respect to the inner product $\langle\cdot, \cdot\rangle_{*}$, the spaces $\mathcal{V}^{j}$ and $\mathcal{V}^{j+1}$ become Hilbert spaces, with the corresponding multi-weighted norm $\|F\|_{*}=\langle F, F\rangle_{*}^{1 / 2}$. Let $\mathcal{W}^{j}$ denote the orthogonal complement with respect to $\langle\cdot, \cdot\rangle_{*}$ of the coarse space $\mathcal{V}^{j}$ in the fine space $\mathcal{V}^{j+1}$, so that

$$
\mathcal{V}^{j+1}=\mathcal{V}^{j} \oplus \mathcal{W}^{j},
$$

and therefore

$$
\mathcal{V}^{j+1}=\mathcal{V}^{0} \oplus \mathcal{W}^{0} \oplus \mathcal{W}^{1} \oplus \cdots \oplus \mathcal{W}^{j}
$$


The spaces $\mathcal{W}^{j}$ are called the wavelet spaces and its nonzero elements are called wavelets. The dimension of $\mathcal{W}^{j}$ is $\left|V^{j+1}\right|-\left|V^{j}\right|=\left|E^{j}\right|$.

In this section we construct a basis for $\mathcal{W}^{j}$, consisting of wavelets of small support. Here we follow [5] and [6].

To a vertex $u \in V^{j+1} \backslash V^{j}$, which is a midpoint of some edge $\left[a_{1} a_{2}\right] \in E^{j}$, we associate a wavelet $\psi_{u}^{j}$ in the following way. We take

$$
\psi_{u}^{j}(\boldsymbol{\eta})=\sigma_{a_{1}, u}^{j}(\boldsymbol{\eta})+\sigma_{a_{2}, u}^{j}(\boldsymbol{\eta}), \quad \text { for } \boldsymbol{\eta} \in \mathbb{S}^{2},
$$

where the functions $\sigma_{a_{1}, u}^{j}$ and $\sigma_{a_{2}, u}^{j}$ are called semi-wavelets. They are taken as

$$
\begin{aligned}
& \sigma_{a_{1}, u}^{j}(\boldsymbol{\eta})=s_{a_{1}} \varphi_{a_{1}}^{j+1}(\boldsymbol{\eta})+\sum_{w \in V_{a_{1}}^{j+1}} s_{w} \varphi_{w}^{j+1}(\boldsymbol{\eta}), \text { with } s_{a_{1}}, s_{w} \in \mathbb{R}, w \in V_{a_{1}}^{j+1}, \\
& \sigma_{a_{2}, u}^{j}(\boldsymbol{\eta})=s_{a_{2}} \varphi_{a_{2}}^{j+1}(\boldsymbol{\eta})+\sum_{w \in V_{a_{2}}^{j+1}} t_{w} \varphi_{w}^{j+1}(\boldsymbol{\eta}), \text { with } s_{a_{2}}, t_{w} \in \mathbb{R}, w \in V_{a_{2}}^{j+1}
\end{aligned}
$$

and satisfy the conditions

$$
\left\langle\varphi_{w}^{j}, \sigma_{a_{1}, u}^{j}\right\rangle_{*}=\left\{\begin{aligned}
-2^{-2 j} \gamma & \text { if } w=a_{1}, \\
2^{-2 j} \gamma & \text { if } w=a_{2}, \\
0 & \text { otherwise }
\end{aligned}\right.
$$

for a given $\gamma \neq 0$. This means that $\sigma_{a_{1}, u}^{j}$ is orthogonal to all nodal functions from the level $j$, except for $\varphi_{a_{1}}^{j}$ and $\varphi_{a_{2}}^{j}$ and its support is included in $\mathcal{M}_{a_{1}}^{j}$. From (8) we find that

$$
\left\langle\varphi_{w}^{j}, \psi_{u}^{j}\right\rangle_{*}=\left\langle\varphi_{w}^{j}, \sigma_{a_{1}, u}\right\rangle_{*}+\left\langle\varphi_{w}^{j}, \sigma_{a_{2}, u}\right\rangle_{*}=0 \quad \text { for all } w \in V^{j},
$$

and therefore $\psi_{u}^{j}$ belongs to the wavelet space $\mathcal{W}^{j}$, being orthogonal to a basis of $\mathcal{V}^{j}$. The support of $\psi_{u}^{j}$ is included in $\mathcal{M}_{a_{1}}^{j} \cup \mathcal{M}_{a_{2}}^{j}$.

Suppose the degree (the number of neighbors) of $a_{1}$ is $s_{1}:=\left|V_{a_{1}}^{j}\right|=\left|V_{a_{1}}^{j+1}\right|$ and its fine neighbors are $b_{0}, b_{1}, \ldots b_{s_{1}-1}$, with $b_{0}=u$, the degree of $a_{2}$ is $s_{2}$ and its fine neighbors are $c_{0}, c_{1}, \ldots c_{s_{2}-1}$, with $c_{0}=u$. A choice for the coefficients $s_{a_{1}}, s_{b_{i}}, s_{a_{2}}$ and $t_{c_{i}}$ is

$$
\begin{aligned}
& s_{a_{1}}=-\frac{3}{2 s_{1}}, \quad s_{b_{i}}=\frac{3}{28 s_{1}}+\theta\left(i, s_{1}\right), \quad i=0,1, \ldots, s_{1}-1, \\
& s_{a_{2}}=-\frac{3}{2 s_{2}}, \quad t_{c_{i}}=\frac{3}{28 s_{2}}+\theta\left(i, s_{2}\right), \quad i=0,1, \ldots, s_{2}-1,
\end{aligned}
$$

where $\theta(i, s)=\frac{\lambda^{i}+\lambda^{s-i}}{\sqrt{21}\left(1-\lambda^{s}\right)}$, with $\lambda=(-5+\sqrt{21}) / 2$.

This choice was made for planar triangulations in Lemma 3.3 of [6]. Similarly we can prove the result for our triangulations, due to Lemma 1. Note that the coefficients do not depend on the level $j$.

Now we have obtained the set $\left\{\psi_{u}^{j}, u \in V^{j+1} \backslash V^{j}\right\}$ of locally supported functions satisfying the orthogonality conditions (9). In order to be a basis for $\mathcal{W}^{j}$, the wavelets $\psi_{u}^{j}, u \in V^{j+1} \backslash V^{j}$ must be linearly independent for every $j \in \mathbb{N}$. The linear independence can be proved in the same way as in [6].

To be useful in practice the basis of wavelets should be dense in $C\left(\mathbb{S}^{2}\right)$ (and therefore in $\left.L^{2}\left(\mathbb{S}^{2}\right)\right)$ and should satisfy the Riesz stability property. The set

$$
\bigcup_{j \geq 0} \mathcal{V}^{j}=\mathcal{V}^{0} \oplus \bigoplus_{j=0}^{\infty} \mathcal{W}^{j}
$$


is indeed dense in $C\left(\mathbb{S}^{2}\right)$, which follows immediately from the density of

$$
\bigcup_{j \geq 0}\left\{\phi_{u}^{j}: u \in V^{j}\right\}
$$

in $C(\Omega)$ and from Lemma 5 and Lemma 6 .

Now we must establish that the set

$$
\left\{\varphi_{u}^{0}: u \in V^{0}\right\} \cup \bigcup_{j=0}^{\infty}\left\{2^{j} \psi_{u}^{j}: u \in V^{j+1} \backslash V^{j}\right\}
$$

forms a Riesz basis of the space given in (10). This is equivalent to the existence of positive constants $R_{1}, R_{2}$, independent of the level $j$, such that for all sets of real coefficients $\left\{d_{u}^{j}, j \geq-1, u \in V^{j+1} \backslash V^{j}\right\}$ with

$$
\sum_{j \geq-1} \sum_{u \in V^{j+1} \backslash V^{j}}\left(d_{u}^{j}\right)^{2}<\infty
$$

the inequalities

$$
\begin{aligned}
R_{1} \sum_{j \geq-1} \sum_{u \in V^{j+1} \backslash V^{j}}\left(d_{u}^{j}\right)^{2} & \leq\left\|\sum_{j \geq-1} \sum_{u \in V^{j+1} \backslash V^{j}} d_{u}^{j} 2^{j} \psi_{u}^{j}\right\|_{L^{2}\left(\mathbb{S}^{2}\right)}^{2} \\
& \leq R_{2} \sum_{j \geq-1} \sum_{u \in V^{j+1} \backslash V^{j}}\left(d_{u}^{j}\right)^{2},
\end{aligned}
$$

hold with the notation $\psi_{u}^{-1}=2 \varphi_{u}^{0}$ and $V^{-1}=\emptyset$.

Using Theorem 5.1 from [6], we establish that

$C_{1} \sum_{j \geq-1} \sum_{u \in V^{j+1} \backslash V^{j}}\left(d_{u}^{j}\right)^{2} \leq\left\|\sum_{j \geq-1} \sum_{u \in V^{j+1} \backslash V^{j}} d_{u}^{j} 2^{j} \psi_{u}^{j}\right\|_{*}^{2} \leq C_{2} \sum_{j \geq-1} \sum_{u \in V^{j+1} \backslash V^{j}}\left(d_{u}^{j}\right)^{2}$

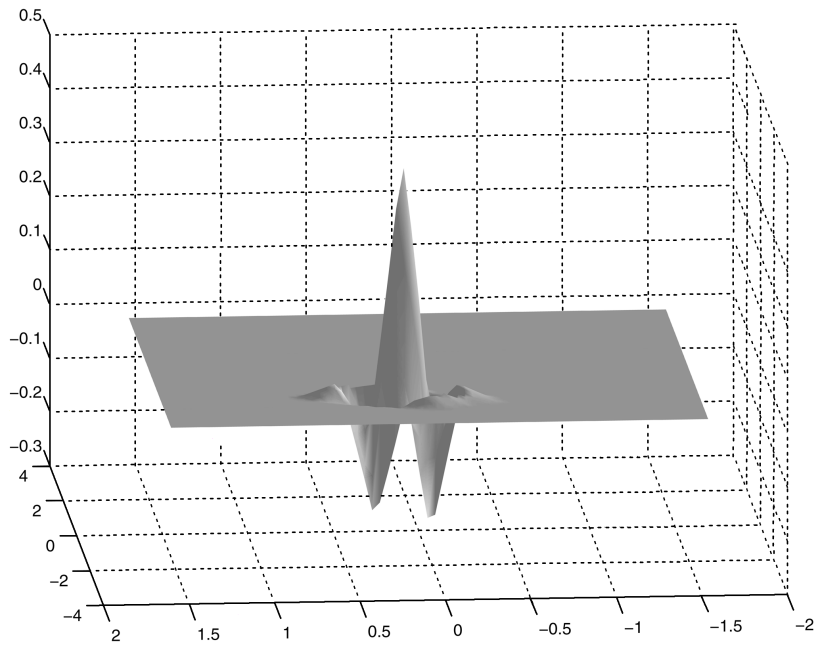

Figure 2. A wavelet $\psi_{u}^{j}$ represented in spherical coordinates. 


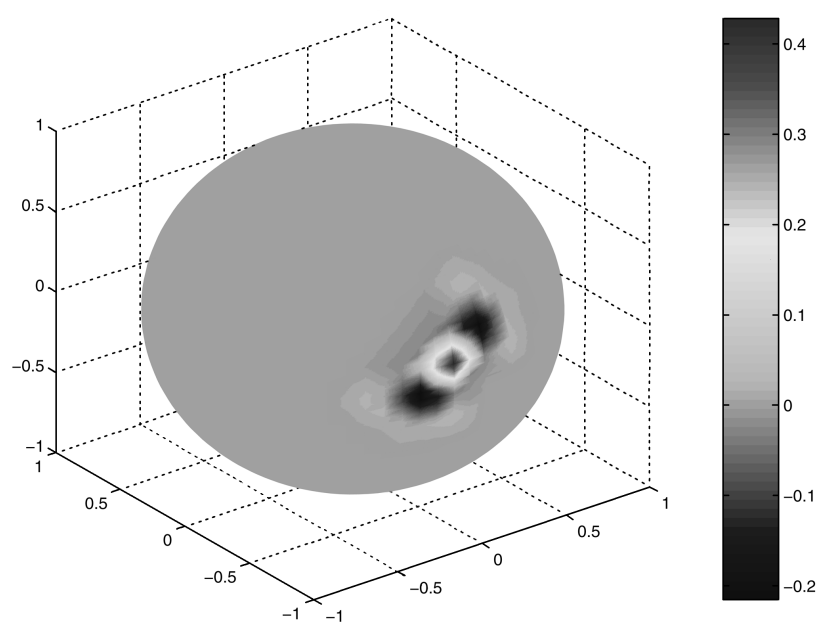

FiguRE 3. A wavelet $\psi_{u}^{j}$ represented on the sphere.

with $C_{1}=L_{B}^{2} / 4$ and $C_{2}=25 / 21 t_{0}$, where $L_{B}=0.0468962 \ldots$ and $t_{0}=$ $\max \left(6, \max _{w \in V^{0}} t(w)\right), t(w)$ denoting, as before, the number of neighbors of the vertex $w$.

If we want Riesz bounds for $\|\cdot\|_{L^{2}\left(\mathbb{S}^{2}\right)}$, we can use Lemma 5 and obtain

$$
\begin{aligned}
\frac{C_{1}}{M} \sum_{j \geq-1} \sum_{u \in V^{j+1} \backslash V^{j}}\left(d_{u}^{j}\right)^{2} & \leq\left\|\sum_{j \geq-1} \sum_{u \in V^{j+1} \backslash V^{j}} d_{u}^{j} 2^{j} \psi_{u}^{j}\right\|_{L^{2}\left(\mathbb{S}^{2}\right)}^{2} \\
& \leq \frac{C_{2}}{m} \sum_{j \geq-1} \sum_{u \in V^{j+1} \backslash V^{j}}\left(d_{u}^{j}\right)^{2} .
\end{aligned}
$$

Figures 2 and 3 show a wavelet $\psi_{u}^{j}$ represented in spherical coordinates and on the sphere, respectively.

\section{QUASI-INTERPOLANTS}

Following the idea of Oswald (see [14, Chapter 2) we will build a piecewise linear quasi-interpolant $Q: L^{2}(\Omega) \rightarrow S$, where $S$ is one of the spaces $S^{j}$ defined in Section 3, For each space $S^{j}$, a quasi-interpolant $Q^{j}$ is defined accordingly. For simplicity we present the construction only for the space $S^{0}=S$ and we study the error $\|f-Q f\|_{L^{2}(\Omega)}$ for $f \in L^{2}(\Omega)$.

Consider an arbitrary vertex $P_{i}$ of a triangle of $\mathcal{T}^{0}=\mathcal{T}$ of $\Omega$ and fix a triangle $\Delta_{i}$ of $\mathcal{T}$ which is at a distance $\leq c \overline{h_{i}}$ from $P_{i}$, with the constant $c$ independent of $i$. Here $\overline{h_{i}}$ is the diameter of the support of the nodal function $\phi_{P_{i}}$. Due to the regularity of the triangulation $\mathcal{T}^{0}$, the diameter of $\Delta_{i}$ satisfies $h\left(\Delta_{i}\right) \approx \overline{h_{i}}$. Let $M_{i n}$, $n=0,1,2$, and $\lambda_{i n}(P), n=0,1,2,3$, denote the vertices of $\Delta_{i}$ and the barycentric coordinates of a point $P \in \mathbb{R}^{3}$ with respect to $O M_{i 0} M_{i 1} M_{i 2}$, respectively. The functions $\lambda_{i n}$ span the space of linear polynomials in three variables defined on $\Omega$. Another property of the functions $\lambda_{i n}$ is $\lambda_{i 3}=1-\lambda_{i 0}-\lambda_{i 1}-\lambda_{i 2}$ and for $(x, y, z)$ belonging to the plane of the triangle $\Delta_{i}$ we have $\lambda_{i 3}(x, y, z)=0$. To get a better 
idea of what the functions $\lambda_{i n}$ look like, we write $\lambda_{i 0}$ as

$$
\lambda_{i 0}(x, y, z)=\left|\begin{array}{ccc}
x & y & z \\
x_{1} & y_{1} & z_{1} \\
x_{2} & y_{2} & z_{2}
\end{array}\right| \cdot\left|\begin{array}{ccc}
x_{0} & y_{0} & z_{0} \\
x_{1} & y_{1} & z_{1} \\
x_{2} & y_{2} & z_{2}
\end{array}\right|^{-1},
$$

where $\left(x_{l}, y_{l}, z_{l}\right), l=0,1,2$, are the coordinates of $M_{i l}$, respectively.

Then a straightforward calculation shows that the functions

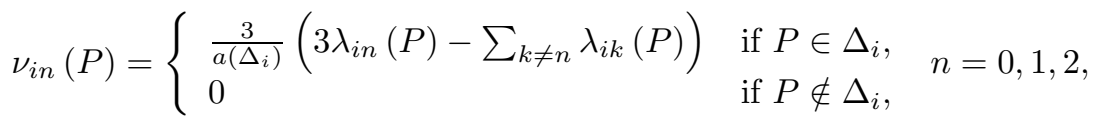

are $L^{2}$-biorthogonal to the basis $\left\{\lambda_{i n}, n=0,1,2\right\}$ of the polynomial space mentioned above. Finally we define the functions

$$
\nu_{i}(P)=\sum_{n=0}^{2} \lambda_{i n}\left(P_{i}\right) \nu_{i n}(P)
$$

with the support $\Delta_{i}$. The quasi-interpolant is now defined as

$$
Q f(P)=\sum_{i}\left(\int_{\Delta_{i}} f \nu_{i} d x\right) \phi_{P_{i}}(P) .
$$

This quasi-interpolant satisfies the following properties:

Proposition 7. For every triangle $K$ of $\Omega$ we have

$$
\|Q f\|_{L^{2}(K)} \leq C\|f\|_{L^{2}(\bar{K})},
$$

where $\bar{K}$ denotes the union of $K$ and all its neighboring triangles which have a distance from $K$ less than or equal to $c \cdot h(K)$.

Proof. The proof follows the same ideas as in [14], Chapter 2.

Proposition 8. The quasi-interpolant $Q$ preserves linear polynomials.

Proof. Let us take a nonconstant linear polynomial $q$ defined on $\Omega$. Using the barycentric coordinates with respect to any of the simplices $O M_{i 0} M_{i 1} M_{i 2}$, we can write

$$
q(P)=\sum_{n=0}^{2} \alpha_{i n} \lambda_{i n}(P)
$$

with some coefficients $\alpha_{i n}$ and evaluate $Q q$ at an arbitrary vertex $P_{i}$, as

$$
\begin{aligned}
Q q\left(P_{i}\right) & =\int_{\Delta_{i}} q \nu_{i} d x=\int_{\Delta_{i}} \sum_{n=0}^{2} \alpha_{i n} \lambda_{i n} \nu_{i} d x \\
& =\sum_{n=0}^{2} \alpha_{i n} \int_{\Delta_{i}} \lambda_{i n} \nu_{i} d x=\sum_{n=0}^{2} \alpha_{i n} \lambda_{i n}\left(P_{i}\right)=q\left(P_{i}\right),
\end{aligned}
$$

by using the biorthogonality of the sets $\left\{\lambda_{i n}, n=0,1,2\right\}$ and $\left\{\nu_{i n}, n=0,1,2\right\}$. 
Thus for any triangle $K$ of $\Omega$ we have local preservation of linear polynomials in the following sense: if $f$ coincides with a linear polynomial on $\bar{K}$, then $Q f=f$ on $K$. This property is equivalent to $Q p=p$ for every linear polynomial $p$ defined on $\Omega$. Since the constant function $f \equiv 1$ belongs to the space $S^{0}$, we have that $Q p=p$ for every polynomial of degree at most 1 , so we can say that $Q$ is a quasi-interpolant of order 1.

Note that the previous two propositions remain valid for the whole sequence of subspaces $\left\{S^{j}\right\}_{j \geq 0}$, with the interpolants $Q^{j}$ defined accordingly. Furthermore in Proposition 7 the constant $C$ is independent of the level $j$ (see [14]).

In the following we want to evaluate the error $\|f-Q f\|_{L^{2}(\Omega)}$ by writing the integral of the function defined on $\Omega$ as a sum of double integrals. We need to replace each $Q f: \Delta_{i} \rightarrow \mathbb{R}$ with a two-variable quasi-interpolant $\widetilde{Q} f_{i}: \operatorname{Pr}_{\mathcal{P}} \Delta_{i} \subseteq$ $\mathbb{R}^{2} \rightarrow \mathbb{R}$, where $\mathcal{P}$ is one of the coordinate planes. Moreover we establish conditions under which the quasi-interpolant $\widetilde{Q} f_{i}$ coincides with the quasi-interpolant built by Oswald in 14, Chapter 2, for the two-dimensional case.

5.1. The quasi-interpolant $\widetilde{Q} \widetilde{f}$ for a function $\widetilde{f} \in L^{2}(\widetilde{\Delta}), \widetilde{\Delta} \subseteq \mathbb{R}^{2}$. This construction was carried out by Oswald in 14 for arbitrary dimension.

Let us take a triangulation $\mathcal{D}$ in $\mathbb{R}^{2}$, an arbitrary nodal point $N_{i}\left(\widetilde{\alpha}_{i}, \widetilde{\beta}_{i}\right)$ as a vertex of a triangle of $\mathcal{D}$ and $D_{i}$ a triangle situated at a distance $\leq c \widetilde{h}_{i}$ from $N_{i}$. Denote the vertices of $D_{i}$ by $M_{n}\left(\widetilde{x}_{n}, \widetilde{y}_{n}\right), n=0,1,2$.

Let us define the functions

$$
\widetilde{\lambda}_{i 0}(x, y)=\left|\begin{array}{ccc}
x & y & 1 \\
\widetilde{x}_{1} & \widetilde{y}_{1} & 1 \\
\widetilde{x}_{2} & \widetilde{y}_{2} & 1
\end{array}\right| \cdot\left|\begin{array}{ccc}
\widetilde{x}_{0} & \widetilde{y}_{0} & 1 \\
\widetilde{x}_{1} & \widetilde{y}_{1} & 1 \\
\widetilde{x}_{2} & \widetilde{y}_{2} & 1
\end{array}\right|^{-1}
$$

and analogously $\widetilde{\lambda}_{i 1}$ and $\widetilde{\lambda}_{i 2}$. Then we consider the functions

$$
\begin{gathered}
\widetilde{\nu}_{i n}(P)=\left\{\begin{array}{ll}
\frac{3}{a\left(D_{i}\right)}\left(3 \widetilde{\lambda}_{i n}(P)-\sum_{k \neq n} \widetilde{\lambda}_{i k}(P)\right) & \text { if } P \in D_{i}, \\
0 & \text { if } P \notin D_{i},
\end{array} \quad n=0,1,2,\right. \\
\widetilde{\nu}_{i}(P)=\sum_{n=0}^{2} \widetilde{\lambda}_{i n}\left(N_{i}\right) \widetilde{\nu}_{i n}(P) .
\end{gathered}
$$

The nodal function $\widetilde{\phi}_{N_{i}}(x, y)$ restricted to the triangle $N_{i} N_{j} N_{k}$ of $\mathcal{D}$ is equal to

$$
\left|\begin{array}{ccc}
x & y & 1 \\
\widetilde{\alpha} j & \widetilde{\beta}_{j} & 1 \\
\widetilde{\alpha}_{k} & \widetilde{\beta}_{k} & 1
\end{array}\right| \cdot\left|\begin{array}{ccc}
\widetilde{\alpha}_{i} & \widetilde{\beta}_{i} & 1 \\
\widetilde{\alpha}_{j} & \widetilde{\beta}_{j} & 1 \\
\widetilde{\alpha}_{k} & \widetilde{\beta}_{k} & 1
\end{array}\right|^{-1}
$$

where $\left(\widetilde{\alpha}_{j}, \widetilde{\beta}_{j}\right)$ and $\left(\widetilde{\alpha}_{k}, \widetilde{\beta}_{k}\right)$ are the coordinates of the points $N_{j}$ and $N_{k}$, respectively. The quasi-interpolant $\widetilde{Q} \widetilde{f}$ has a similar expression to (12), namely

$$
\widetilde{Q} \widetilde{f}(P)=\sum_{i}\left(\int_{D_{i}} \widetilde{f}_{\widetilde{\nu}_{i}} d x\right) \widetilde{\phi}_{N_{i}}(P), P \in \widetilde{\Delta} .
$$


The first term of the sum (13) is

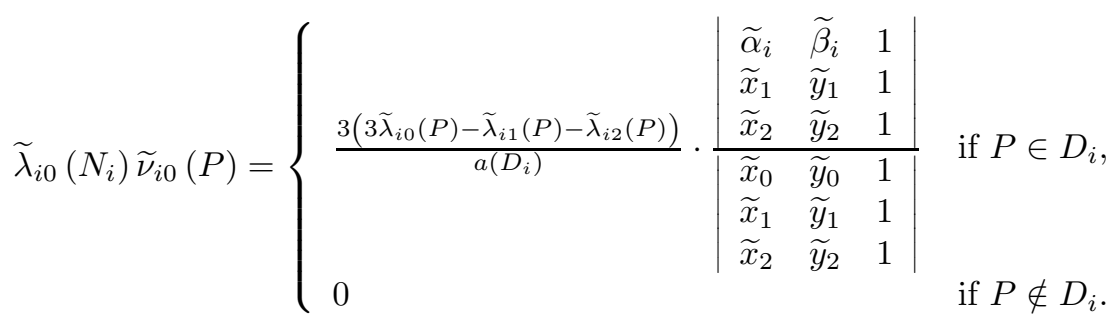

5.2. Projecting the polyhedral surface $\Omega$ onto the coordinate planes. Let us divide the polyhedral surface $\Omega$ into six parts: $\Omega=\Omega_{1} \cup \Omega_{2} \cup \Omega_{3} \cup \Omega_{4} \cup \Omega_{5} \cup \Omega_{6}$ according to the following rule: first we split the sphere $\mathbb{S}^{2}$ into six equal disjoint parts $\mathcal{S}_{m}, m=1, \ldots, 6$, building the inscribed cube with faces parallel to the coordinate axes and arches of big circles between the neighbor vertices of the cube. The parts $\mathcal{S}_{1}$ and $\mathcal{S}_{2}$ will be symmetric with respect to the plane $x O y, \mathcal{S}_{3}$ and $\mathcal{S}_{4}$ will be symmetric with respect to the plane $y O z$, resp. $\mathcal{S}_{5}$ and $\mathcal{S}_{6}$ will be symmetric with respect to the plane $z O x$. The intersection of two neighbors $\mathcal{S}_{m}$ will be the big arch between the corresponding two vertices of the cube.

Thus, $\Omega_{m}$ will contain those triangles of $\Omega$ that have all the vertices situated in $\mathcal{S}_{m}$. There will also be triangles that have the vertices situated in different parts $\mathcal{S}_{m}$. In order to decide to which $\Omega_{m}$ such triangles belong, we measure for each of them the angles $\alpha_{l}, l=1,2,3$, between its plane and the planes $x O y, y O z$ and $z O x$, respectively, and then take the minimum of $\alpha_{l}$. If for example the minimum is attained for $l=1$, then the triangle will belong to either $\Omega_{1}$ or $\Omega_{2}$, depending on which $\Omega_{m}$ contains one vertex of the triangle. In the beginning we choose the triangulation (the polyhedron) such that no triangle has vertices situated on the "opposite" $\Omega_{m}$.

Now let us reorder the triangles of the triangulation $\mathcal{T}$ so that the part $\Omega_{m}$ contains the triangles $T_{i}^{m}, i=1, \ldots, k_{m}, m=1, \ldots, 6$.

We have to make some remarks concerning the interpolant $Q$. For the point $P_{i} \in \Omega_{m}$, it is possible for the associated triangle $\Delta_{i}$ not to belong to $\Omega_{m}$. This is why we ask for all the associated triangles $\Delta_{i}$ to be chosen so that they contain the vertices $P_{i}$, respectively. We will see in Proposition 9 that we need this condition for our purposes anyway.

Without loss of generality, we restrict ourselves to the part $\Omega_{1}$ consisting of the triangles $T_{i}^{1}, i=1, \ldots, k_{1}$.

Now the part $\Omega_{1}$ is split into three zones $\Omega_{1}=\Omega_{1}^{\mathbf{x}} \cup \Omega_{1}^{\mathbf{y}} \cup \Omega_{1}^{\mathbf{z}}$ according to the following rule. For each triangle $T_{i}^{1}$ of the part $\Omega_{1}$, we calculate the angles between its plane and the coordinate planes. Then we project $T_{i}^{1}$ onto that coordinate plane that gives the minimum angle. If there is more than one angle that attains the minimum, we perform only one of the projections.

To do this, we evaluate the quantities $\left|\mathcal{A}_{\text {xoy }} / \mathcal{A}\right|,\left|\mathcal{A}_{\text {yoz }} / \mathcal{A}\right|$ and $\left|\mathcal{A}_{z o x} / \mathcal{A}\right|$, defined in Lemma 1, representing the absolute values of the cosines of the respective angles. Then we choose the maximum of these quantities. The parts $\Omega_{1}^{\mathbf{x}}, \Omega_{1}^{\mathbf{y}}$ and $\Omega_{1}^{\mathbf{z}}$ contain those triangles that project onto $y O z, z O x$ and $x O y$, respectively. We reorder the triangles of $\Omega_{1}$ such that the zone $\Omega_{1}^{\mathbf{x}}$ contains the triangles $T \mathbf{x}_{i}^{1}, i=1, \ldots, k_{1}^{\mathbf{x}}$, and analogously for $\Omega_{1}^{\mathbf{y}}$ and $\Omega_{1}^{\mathbf{z}}$. Obviously $k_{1}^{\mathbf{x}}+k_{1}^{\mathbf{y}}+k_{1}^{\mathbf{z}}=k_{1}$. 
We need to consider "enlarged" regions $\overline{\Omega_{1}^{\mathbf{x}}}, \overline{\Omega_{1}^{\mathbf{y}}}$ and $\overline{\Omega_{1}^{\mathbf{z}}}$ for our quasi-interpolant. The region $\overline{\Omega_{1}^{\mathbf{x}}}$ consists of the union of the triangles $T \mathbf{x}_{i}^{1}$ and all their neighboring triangles from $\Omega$, where we consider two triangles as neighbors if they share a common vertex. The regions $\overline{\Omega_{1}^{\mathrm{y}}}$ and $\overline{\Omega_{1}^{\mathbf{z}}}$ are taken analogously. These enlarged regions are not necessarily connected and they may contain triangles that have degenerated projections onto the respective planes. In this case, we redefine the enlarged zones eliminating the triangles that have degenerated projections.

Again, without loss of generality, we restrict to the part $\Omega_{1}^{\mathbf{z}}$ and try, in the expression of $Q f$, to replace $z$ by $z_{i}(x, y)$ from the equation of the plane of each triangle $T \mathbf{z}_{i}^{1}, i=1, \ldots, k_{1}^{\mathbf{z}}$. In this case $(x, y) \in \operatorname{Pr}_{\text {xoy }} T \mathbf{z}_{i}^{1}$ and the surface element $d T \mathbf{z}_{i}^{1}$ equals $\frac{a\left(T \mathbf{z}_{i}^{1}\right)}{a\left(\operatorname{Pr}_{x o y} T \mathbf{z}_{i}^{1}\right)} d x d y$.

Moreover it is easy to show that $\lambda_{i n}\left(x, y, z_{i}(x, y)\right)=\widetilde{\lambda}_{i n}(x, y)$ for $(x, y)$ belonging to the triangle $D_{i}$, which is the projection of $T \mathbf{z}_{i}^{1}$ onto $x O y$. Let us now compare the nodal functions. Take the face $\left(A_{i} A_{j} A_{k}\right)$ of $\overline{\Omega_{1}^{\mathbf{z}}}$ with $A_{i}\left(\alpha_{i}, \beta_{i}, \gamma_{i}\right), A_{j}\left(\alpha_{j}, \beta_{j}, \gamma_{j}\right)$, $A_{k}\left(\alpha_{k}, \beta_{k}, \gamma_{k}\right)$. The equation of this face is

$$
\left|\begin{array}{cccc}
x & y & z & 1 \\
\alpha_{i} & \beta_{i} & \gamma_{i} & 1 \\
\alpha_{j} & \beta_{j} & \gamma_{j} & 1 \\
\alpha_{k} & \beta_{k} & \gamma_{k} & 1
\end{array}\right|=0
$$

or $\mathcal{A}_{\text {yoz }} x+\mathcal{A}_{z o x} y+\mathcal{A}_{x o y} z-\xi=0$. The nodal function $\phi_{A_{i}}$, restricted to the triangle $A_{i} A_{j} A_{k}$, is equal to

$$
\left|\begin{array}{ccc}
x & y & z \\
\alpha_{j} & \beta_{j} & \gamma_{j} \\
\alpha_{k} & \beta_{k} & \gamma_{k}
\end{array}\right| \cdot\left|\begin{array}{ccc}
\alpha_{i} & \beta_{i} & \gamma_{i} \\
\alpha_{j} & \beta_{j} & \gamma_{j} \\
\alpha_{k} & \beta_{k} & \gamma_{k}
\end{array}\right|^{-1} .
$$

Furthermore, by replacing $z$ using the equation $\mathcal{A}_{\text {yoz }} x+\mathcal{A}_{z o x} y+\mathcal{A}_{\text {xoy }} z-\xi=0$ and making some column transformations, we obtain

$$
\begin{aligned}
\phi_{A_{i}}(x, y, z(x, y)) & =\left|\begin{array}{ccc}
x & y & \frac{\xi}{\mathcal{A}_{x o y}} \\
\alpha_{j} & \beta_{j} & \frac{\xi}{\mathcal{A}_{x o y}} \\
\alpha_{k} & \beta_{k} & \frac{\xi}{\mathcal{A}_{x o y}}
\end{array}\right| \cdot\left|\begin{array}{ccc}
\alpha_{i} & \beta_{i} & \frac{\xi}{\mathcal{A}_{x o y}} \\
\alpha_{j} & \beta_{j} & \frac{\xi}{\mathcal{A}_{x o y}} \\
\alpha_{k} & \beta_{k} & \frac{\xi}{\mathcal{A}_{x o y}}
\end{array}\right|^{-1} \\
& =\left|\begin{array}{ccc}
x & y & 1 \\
\alpha_{j} & \beta_{j} & 1 \\
\alpha_{k} & \beta_{k} & 1
\end{array}\right| \cdot\left|\begin{array}{ccc}
\alpha_{i} & \beta_{i} & 1 \\
\alpha_{j} & \beta_{j} & 1 \\
\alpha_{k} & \beta_{k} & 1
\end{array}\right|^{-1},
\end{aligned}
$$

which is exactly the nodal function $\widetilde{\phi}_{i}(x, y)$ restricted to the triangle $A_{i}^{\prime} A_{j}^{\prime} A_{k}^{\prime}$ - the projection of the triangle $A_{i} A_{j} A_{k}$ onto $x O y$.

In order to compare the two quasi-interpolants, we need to state the first term of the sum (11) explicitly as

$$
\lambda_{i 0}\left(A_{i}\right) \nu_{i 0}(P)=\left\{\begin{array}{l}
\frac{3\left(3 \lambda_{i 0}(P)-\lambda_{i 1}(P)-\lambda_{i 2}(P)\right)}{a\left(\Delta_{i}^{\left.\frac{1}{2}\right)}\right.} \cdot \frac{\left|\begin{array}{lll}
\alpha_{i} & \beta_{i} & \gamma_{i} \\
x_{1} & y_{1} & z_{1} \\
x_{2} & y_{2} & z_{2}
\end{array}\right|}{\left|\begin{array}{lll}
x_{0} & y_{0} & z_{0} \\
x_{1} & y_{1} & z_{1} \\
x_{2} & y_{2} & z_{2}
\end{array}\right|} \\
0
\end{array}\right.
$$


Comparing the formulae (12) and (14), we can establish the following result.

Proposition 9. Let $Q f$ be the quasi-interpolant given in (12) and $\widetilde{Q} f_{\mathbf{z}}$ the twodimensional quasi-interpolant given in (14), associated to the triangulation obtained by projecting $\overline{\Omega_{1}^{\mathrm{z}}}$ onto $x O y$. If

(1) each point $P_{i}$ is one of the vertices of the triangle $\Delta_{i}^{1}$,

(2) the associated triangles $\Delta_{i}^{1}$ are chosen such that they have non-degenerated projection onto $\mathrm{xOy}$,

then for all $(x, y) \in \operatorname{Pr}_{x o y} \overline{\Omega_{1}^{\mathbf{z}}}$ we have

$$
\begin{aligned}
\widetilde{Q} f_{1}(x, y) & =\sum_{i} \frac{1}{a\left(D_{i}\right)} \cdot G_{i}\left(f_{\mathbf{z}} ; x, y\right), \\
Q f(x, y, \mathbf{z}(x, y)) & =\sum_{i} \frac{1}{a\left(\operatorname{Pr}_{x o y} \Delta_{i}^{1}\right)} \cdot G_{i}\left(f_{\mathbf{z}} ; x, y\right),
\end{aligned}
$$

where the function $\mathbf{z}(x, y)$ is the function whose restrictions to each triangle $\operatorname{Pr}_{\text {xoy }} \overline{T \mathbf{z}_{i}^{1}}\left(\overline{T \mathbf{z}_{i}^{1}}\right.$ any triangle of $\left.\overline{\Omega_{1}^{\mathbf{z}}}\right)$ are equal to the function $z_{i}(x, y)$ which expresses $z$ using the equation of the plane of $\overline{T \mathbf{z}_{i}^{1}}$. Also $f$ and $f_{\mathbf{z}}$ are related by the formula $f\left(x, y, z_{i}(x, y)\right)=f_{\mathbf{z}}(x, y)$, where $(x, y) \in \operatorname{Pr}_{x o y} \overline{T \mathbf{z}_{i}^{1}}$.

Proof. The proof follows immediately from the above calculations and from the fact that, if $P_{i}$ is a vertex of the triangle $\Delta_{i}^{1}$, then we have

$$
\begin{aligned}
& \left|\begin{array}{lll}
\alpha_{i} & \beta_{i} & 1 \\
x_{1} & y_{1} & 1 \\
x_{2} & y_{2} & 1
\end{array}\right| \cdot\left|\begin{array}{lll}
x_{0} & y_{0} & 1 \\
x_{1} & y_{1} & 1 \\
x_{2} & y_{2} & 1
\end{array}\right|^{-1} \\
& \quad=\left|\begin{array}{lll}
\alpha_{i} & \beta_{i} & \gamma_{i} \\
x_{1} & y_{1} & z_{1} \\
x_{2} & y_{2} & z_{2}
\end{array}\right|\left|\begin{array}{lll}
x_{0} & y_{0} & z_{0} \\
x_{1} & y_{1} & z_{1} \\
x_{2} & y_{2} & z_{2}
\end{array}\right|^{-1}
\end{aligned}
$$

together with the other two symmetric equalities for the second and third terms of the sums (11) and (13). If $P_{i}$ is one of the vertices of the triangle $\Delta_{i}^{1}$, then the conclusion follows. We will not write here the expressions for the functions $G_{i}$. The point is that the same functions $G_{i}$ appear on the right sides of the equalities (15) and (16) and so we can relate the two quasi-interpolants.

Now let us turn back to the error $\|f-Q f\|_{L^{2}(\Omega)}$, which can be written as

$$
\begin{aligned}
\|f-Q f\|_{L^{2}(\Omega)}^{2} & =\sum_{m=1}^{6}\|f-Q f\|_{L^{2}\left(\Omega_{m}\right)}^{2} \\
& =\sum_{m=1}^{6} \sum_{i=1}^{k_{m}} \int_{T_{i}^{m}}|f(x, y, z)-Q f(x, y, z)|^{2} d T_{i}^{m} .
\end{aligned}
$$


Projecting now onto the coordinate planes, we obtain

$$
\begin{aligned}
\| f- & Q f \|_{L^{2}(\Omega)}^{2} \\
= & \sum_{m=1}^{2} \sum_{i=1}^{k_{m}^{z}} \int_{\operatorname{Pr}_{x o y} T \mathbf{z}_{i}^{m}} \mid f\left(x, y, z_{i m}(x, y)\right) \\
& -\left.Q f\left(x, y, z_{i m}(x, y)\right)\right|^{2} \frac{a\left(T \mathbf{z}_{i}^{m}\right)}{a\left(\operatorname{Pr}_{x o y} T \mathbf{z}_{i}^{m}\right)} d x d y \\
& +\sum_{m=3}^{4} \sum_{i=1}^{k_{m}^{\mathbf{x}}} \int_{\operatorname{Pr}_{y o z} T \mathbf{x}_{i}^{m}} \mid f\left(x_{i m}(y, z), y, z\right) \\
& +\sum_{m=5}^{6} \sum_{i=1}^{k_{m}^{\mathbf{y}}} \int_{\operatorname{Pr}_{z o x} T \mathbf{y}_{i}^{m}} \mid f\left(x, y_{i m}(z, x), z\right) \\
& \left.-\left.Q f\left(x, x_{i m}(y, z), y, z\right)\right|^{2} \frac{a\left(T \mathbf{x}_{i}^{m}\right)}{a\left(\operatorname{Pr}_{y o z} T \mathbf{x}_{i}^{m}\right)} d y d z, z\right)\left.\right|^{2} \frac{a\left(T \mathbf{y}_{i}^{m}\right)}{a\left(\operatorname{Pr}_{z o x} T \mathbf{y}_{i}^{m}\right)} d z d x
\end{aligned}
$$

and furthermore, using the $L^{2}$-norm on the projected surfaces and Proposition 9 ,

$$
\begin{aligned}
\|f-Q f\|_{L^{2}(\Omega)}^{2}=\sum_{m=1}^{6}\left(\sum_{i=1}^{k_{m}^{\mathbf{z}}}\right. & \frac{a\left(T \mathbf{z}_{i}^{m}\right)}{a\left(\operatorname{Pr}_{x o y} T \mathbf{z}_{i}^{m}\right)}\left\|f_{\mathbf{z}}-\widetilde{Q} f_{\mathbf{z}}\right\|_{L^{2}\left(\operatorname{Pr}_{x o y} T \mathbf{z}_{i}^{n}\right)}^{2} \\
& +\sum_{i=1}^{k_{m}^{\mathbf{x}}} \frac{a\left(T \mathbf{x}_{i}^{m}\right)}{a\left(\operatorname{Pr}_{y o z} T \mathbf{x}_{i}^{m}\right)}\left\|f_{\mathbf{x}}-\widetilde{Q} f_{\mathbf{x}}\right\|_{L^{2}\left(\operatorname{Pr}_{y o z} T \mathbf{x}_{i}^{m}\right)}^{2} \\
& \left.+\sum_{i=1}^{k_{m}^{\mathbf{y}}} \frac{a\left(T \mathbf{y}_{i}^{m}\right)}{a\left(\operatorname{Pr}_{z o x} T \mathbf{y}_{i}^{m}\right)}\left\|f_{\mathbf{y}}-\widetilde{Q} f_{\mathbf{y}}\right\|_{L^{2}\left(\operatorname{Pr}_{z o x} T \mathbf{y}_{i}^{m}\right)}^{2}\right) .
\end{aligned}
$$

It is well known that for an arbitrary plane we have the equality $\cos ^{2} \alpha+\cos ^{2} \beta+$ $\cos ^{2} \gamma=1$, where $\alpha, \beta$ and $\gamma$ are the angles of this plane with $x O y, y O z$ and $z O x$, respectively. Since $\max (\cos \alpha, \cos \beta, \cos \gamma) \geq 1 / \sqrt{3}$, we get

$$
\begin{aligned}
1 & \leq \frac{a\left(T \mathbf{z}_{i}^{m}\right)}{a\left(\operatorname{Pr}_{x o y} T \mathbf{z}_{i}^{m}\right)} \leq \sqrt{3}, \text { for all } i=1, \ldots, k_{m}^{\mathbf{z}}, \quad m=1, \ldots, 6, \\
1 & \leq \frac{a\left(T \mathbf{x}_{i}^{m}\right)}{a\left(\operatorname{Pr}_{y o z} T \mathbf{x}_{i}^{m}\right)} \leq \sqrt{3}, \text { for all } i=1, \ldots, k_{m}^{\mathbf{x}}, \quad m=1, \ldots, 6, \\
1 & \leq \frac{a\left(T \mathbf{y}_{i}^{m}\right)}{a\left(\operatorname{Pr}_{z o x} T \mathbf{y}_{i}^{m}\right)} \leq \sqrt{3}, \text { for all } i=1, \ldots, k_{m}^{\mathbf{y}}, \quad m=1, \ldots, 6 .
\end{aligned}
$$

Hence, we obtain

$$
\sum_{m=1}^{6}\left(\mathcal{E}_{\mathbf{z}}^{m}+\mathcal{E}_{\mathbf{x}}^{m}+\mathcal{E}_{\mathbf{y}}^{m}\right) \leq\|f-Q f\|_{L^{2}(\Omega)}^{2} \leq \sqrt{3} \sum_{m=1}^{6}\left(\mathcal{E}_{\mathbf{z}}^{m}+\mathcal{E}_{\mathbf{x}}^{m}+\mathcal{E}_{\mathbf{y}}^{m}\right),
$$


where

$$
\begin{aligned}
\mathcal{E}_{\mathbf{z}}^{m} & =\left\|f_{\mathbf{z}}^{m}-\widetilde{Q} f_{\mathbf{z}}^{m}\right\|_{L^{2}\left(\operatorname{Pr}_{x o y} \Omega_{m}^{\mathbf{z}}\right)}^{2}, \\
\mathcal{E}_{\mathbf{x}}^{m} & =\left\|f_{\mathbf{x}}^{m}-\widetilde{Q} f_{\mathbf{x}}^{m}\right\|_{L^{2}\left(\operatorname{Pr}_{y o z} \Omega_{m}^{\mathbf{x}}\right)}^{2}, \\
\mathcal{E}_{\mathbf{y}}^{m} & =\left\|f_{\mathbf{y}}^{m}-\widetilde{Q} f_{\mathbf{y}}^{m}\right\|_{L^{2}\left(\operatorname{Pr}_{z o x} \Omega_{m}^{\mathbf{y}}\right)}^{2}
\end{aligned}
$$

and

$$
\left\{\begin{array}{l}
f_{\mathbf{z}}^{m}(x, y)=f\left(x, y, z_{i m}(x, y)\right) \text { on each triangle } \operatorname{Pr}_{x o y} T \mathbf{z}_{i}^{m}, \quad i=1, \ldots, k_{m}^{\mathbf{z}}, \\
f_{\mathbf{x}}^{m}(y, z)=f\left(x_{i m}(y, z), y, z\right) \text { on each triangle } \operatorname{Pr}_{y o z} T \mathbf{x}_{i}^{m}, \quad i=1, \ldots, k_{m}^{\mathbf{x}}, \\
f_{\mathbf{y}}^{m}(z, x)=f\left(x, y_{i m}(z, x), z\right) \text { on each triangle } \operatorname{Pr}_{z o x} T \mathbf{y}_{i}^{m}, \quad i=1, \ldots, k_{m}^{\mathbf{y}}
\end{array}\right.
$$

Thus, the problem of studying the error $\|f-Q f\|_{L^{2}(\Omega)}$ has been reduced to the study of two-dimensional errors from [14].

5.3. Jackson and Bernstein inequalities. Let us recall some of the results in [14. To simplify the notation, we restrict ourselves to the case $m=1$ and to the projections onto $x O y$, and we set $\Theta=\operatorname{Pr}_{x o y} \Omega_{1}^{\mathrm{z}}$. Given a subset $M$ of $L^{2}(\Theta)$, consider the best approximation

$$
E_{M}(f)_{2}=\inf _{g \in M}\|f-g\|_{2}, \quad f \in L^{2}(\Theta) .
$$

We are interested in approximations from above for $E_{M}(f)_{2}$, when $M$ coincides with a subspace $\widetilde{S}^{j}$ of piecewise linear continuous functions on the triangles of a triangulation $\mathcal{D}^{j}$ of $\Theta$. The proofs can be found in [14].

Theorem 10 (Jackson-type inequality). There exists a constant $C$ such that

$$
E_{\widetilde{S}^{j}}\left(f_{1}\right)_{2} \leq\left\|f_{1}-\widetilde{Q}^{j} f_{1}\right\|_{2} \leq C \omega_{1}\left(h\left(\mathcal{D}^{j}\right), f_{1}\right)_{2} \text {, for all } f_{1} \in L^{2}(\Theta),
$$

$\omega_{1}(t, f)_{2}$ being the modulus of smoothness of order 1 associated to the function $f$ and the $L^{2}$-norm on $\Theta$ at the point $t>0$.

The following theorem gives inverse estimates for our approximation, i.e. we give estimates for the moduli of smoothness of functions in $L^{2}(\Theta)$.

Theorem 11 (Bernstein-type inequality). There exists a constant $C$ such that for all $j>0$ and $f_{1} \in L^{2}(\Theta)$ we have

$$
\omega_{1}\left(2^{-j}, f_{1}\right)_{2} \leq C 2^{-j}\left(\left\|f_{1}\right\|_{L^{2}(\Theta)}+\sum_{l=0}^{j} 2^{l} E_{\widetilde{S}^{l}}\left(f_{1}\right)_{2}\right) .
$$

5.4. The spherical quasi-interpolant. The step back to the sphere is straightforward. We define the quasi-interpolants $\mathcal{Q}^{j}: L^{2}\left(\mathbb{S}^{2}\right) \rightarrow \mathcal{V}^{j}$ as

$$
\mathcal{Q}^{j} F=Q^{j}(F \circ p) \circ p^{-1} .
$$

Then the error in the norm $\|\cdot\|_{*}$ is

$$
\begin{aligned}
\left\|\mathcal{Q}^{j} F-F\right\|_{*} & =\left\|\left(\mathcal{Q}^{j} F-F\right) \circ p\right\|_{\Omega}=\left\|\left(\mathcal{Q}^{j} F\right) \circ p-F \circ p\right\|_{\Omega} \\
& =\left\|Q^{j}(F \circ p) \circ p^{-1} \circ p-F \circ p\right\|_{\Omega}=\left\|Q^{j}(F \circ p)-F \circ p\right\|_{\Omega} .
\end{aligned}
$$

So the evaluation of the error for the spherical quasi-interpolant reduces to the evaluation of the error for the quasi-interpolant associated to the polyhedron. 


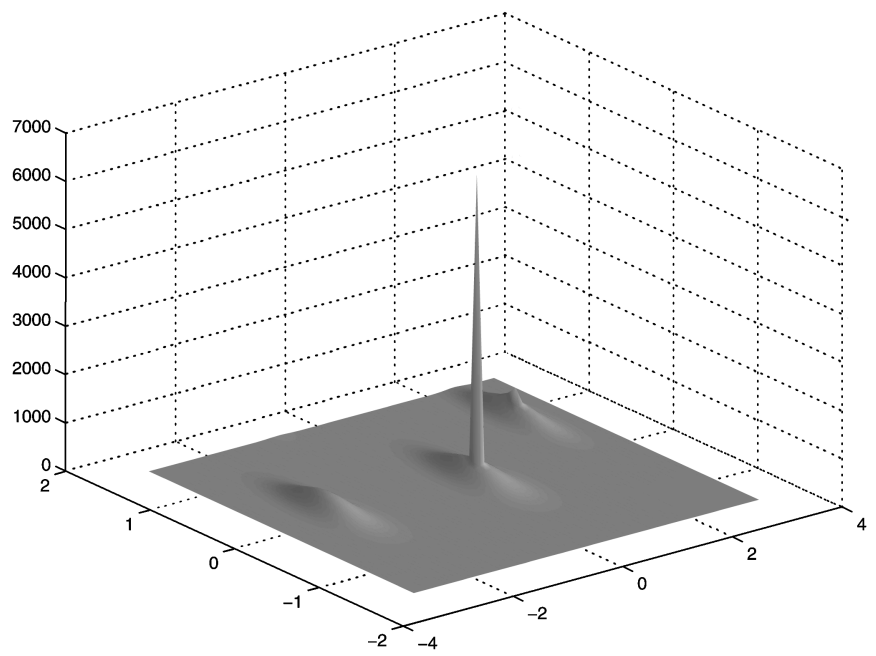

Figure 4. The data set pol5.

\section{NUMERICAL EXAMPLES}

In order to illustrate our wavelets, we take as the initial polyhedron $\Pi$ an octahedron with six vertices and we perform five levels of decomposition. At level five the total number of vertices is 4098 . We consider a particular data set pol5 from the texture analysis of crystals (cf. 20]); see Figure 4. It consists of $36 \times 72$ measurements on the sphere and its main characteristic is that the values over the whole sphere are constant, except for some peaks.

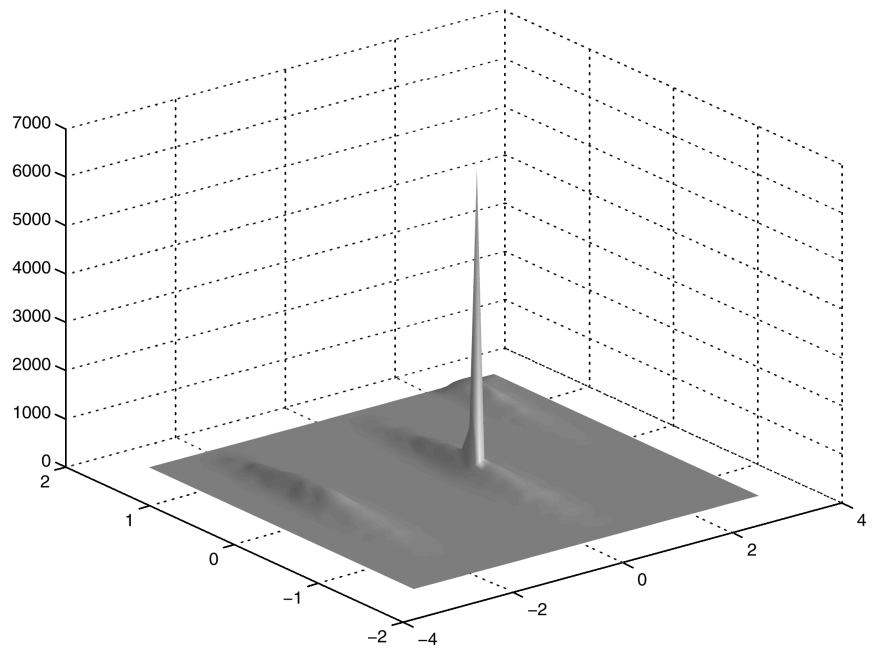

Figure 5. The function $f^{5} \in \mathcal{V}^{5}$, an approximation of pol5 at level 5 . 
First we approximate this data with the function $f^{5} \in \mathcal{V}^{5}$ (see Figure [5). The approximation error

$$
e=\frac{1}{36 \cdot 72} \sum_{i=1}^{36} \sum_{j=1}^{72}\left|f^{5}(i, j)-\operatorname{pol} 5(i, j)\right|
$$

is 1.0984. Since the set $\left\{\varphi_{v}^{j}\right\}_{v \in V^{j}}$ is a basis for $\mathcal{V}^{j}$, for $j=0,1,2, \ldots$, we can write

$$
f^{5}(\boldsymbol{\eta})=\sum_{v \in V^{5}} f_{v}^{5} \varphi_{v}^{5}(\boldsymbol{\eta}), \boldsymbol{\eta} \in \mathbb{S}^{2} .
$$

The vector $\mathbf{f}^{5}=\left(f_{v}^{5}\right)_{v \in V^{5}}$ associated to the function $f^{5}$ was then decomposed into $\mathbf{f}^{0}$ and $\mathbf{g}^{0}, \mathbf{g}^{1}, \mathbf{g}^{2}, \mathbf{g}^{3}, \mathbf{g}^{4}$ using the decomposition algorithm $\mathbf{A} \mathbf{1}^{\prime}$ described in [7], p. 191. The detail coefficients $\mathbf{g}^{j}, j=0, \ldots, 4$, were thresholded to obtain a specific compression rate. More precisely, their components $\left(g_{u}^{j}\right)_{u \in V^{j+1} \backslash V^{j}}$ were replaced by the values $\left(\widehat{g}_{u}^{j}\right)_{u \in V^{j+1} \backslash V^{j}}$ according to a strategy known as hard thresholding. This consists of choosing a threshold $\varepsilon>0$ and then setting

$$
\widehat{g}_{u}^{j}= \begin{cases}g_{u}^{j} & \text { if }\left|g_{u}^{j}\right| \geq \varepsilon \\ 0 & \text { otherwise }\end{cases}
$$

The ratio of the number of nonzero coefficients to the total number,

$$
\frac{\sum_{j=0}^{4}\left|\left\{u \in V^{j+1} \backslash V^{j}: \widehat{g}_{u}^{j} \neq 0\right\}\right|}{\sum_{j=0}^{4}\left|V^{j+1} \backslash V^{j}\right|},
$$

is referred to as the compression rate.

After compression we perform the reconstruction (see the reconstruction algorithm A2' in [7], p. 191), yielding an approximation with error $\mathbf{e}^{5}, \mathbf{e}^{5}=\mathbf{f}^{5}-\widehat{\mathbf{f}}^{5}$, where $\widehat{\mathbf{f}}^{5}=\left(\widehat{f}_{v}^{5}\right)_{v \in V^{5}}$ is the vector associated to the reconstructed function $\widehat{f}^{5}$.

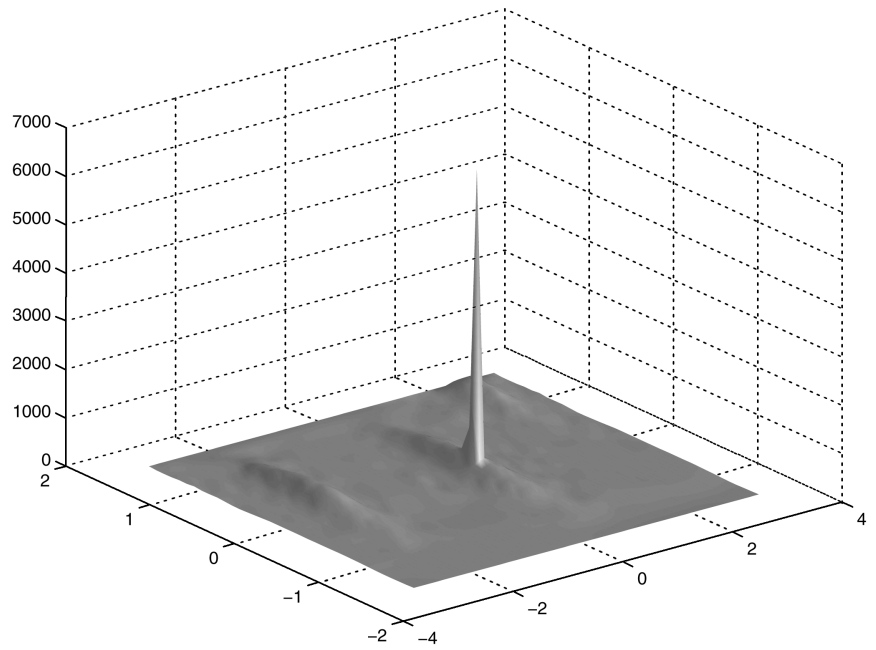

FIGURE 6. The reconstructed function $\widehat{f}^{5}$ for the compression rate 0.1 . 
TABLE 1. Reconstruction errors for some compression rates.

\begin{tabular}{|l|l|r|r|l|}
\hline comp. rate & nr. of zero coeff. & $\left\|\mathbf{e}^{5}\right\|_{\infty}$ & $\left\|\mathbf{e}^{5}\right\|_{2}$ & mean $\left(\mathbf{e}^{5}\right)$ \\
\hline 0.05 & 3888 & 123.09 & 1764.80 & 21.70 \\
0.1 & 3683 & 49.16 & 659.32 & 7.83 \\
0.25 & 3070 & 11.47 & 169.78 & 1.92 \\
0.5 & 2046 & 0.99 & 12.94 & 0.12 \\
0.75 & 1024 & 0.07 & 0.63 & 0.004 \\
\hline
\end{tabular}

We have measured this error in several ways:

- the maximum error given by

$$
\left\|\mathbf{e}^{5}\right\|_{\infty}=\max _{\boldsymbol{\eta} \in \mathbb{S}^{2}}\left|\mathbf{e}^{5}(\boldsymbol{\eta})\right|=\max _{v \in V^{5}}\left|\mathbf{e}^{5}(v)\right| ;
$$

- the 2-norm

$$
\left\|\mathbf{e}^{5}\right\|_{2}=\left(\sum_{v \in V^{5}}\left|f_{v}^{5}-\widehat{f}_{v}^{5}\right|^{2}\right)^{1 / 2} ;
$$

- the mean absolute error over the vertices

$$
\operatorname{mean}\left(\mathbf{e}^{5}\right)=\frac{1}{\left|V^{5}\right|} \sum_{v \in V^{5}}\left|\mathbf{e}^{5}(v)\right|
$$

Figures 6, 7, and 8 show the reconstructed functions $\widehat{f}^{5}$ for different compression rates, and the errors are tabulated in Table 1 .

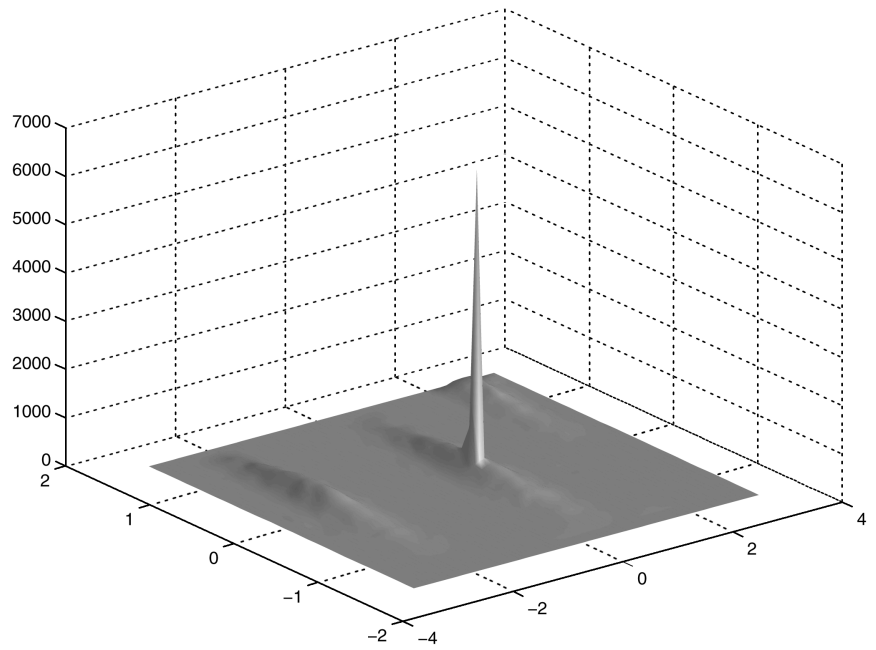

FiguRE 7. The reconstructed function $\widehat{f}^{5}$ for the compression rate 0.25 . 


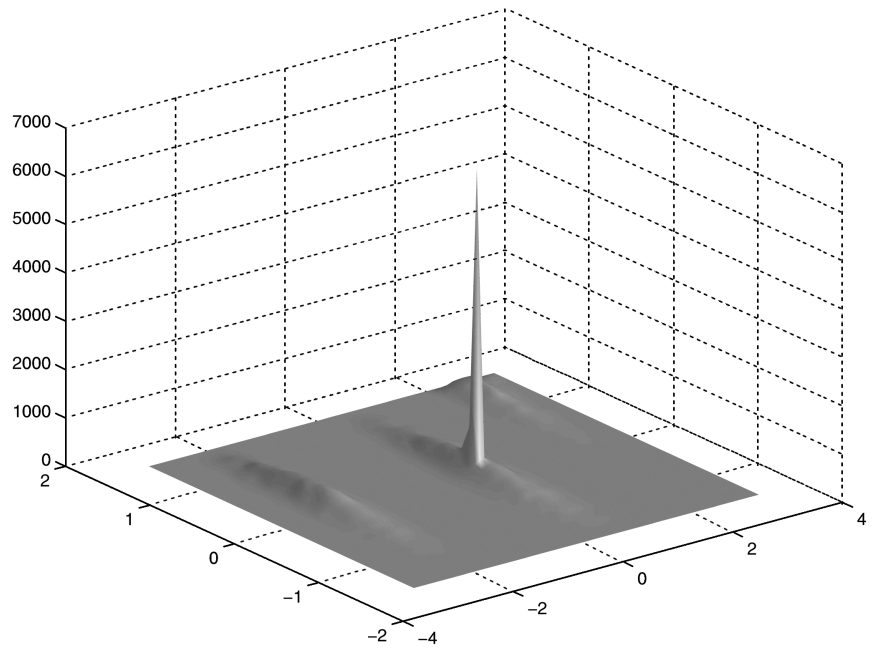

FiguRE 8. The reconstructed function $\widehat{f}^{5}$ for the compression rate 0.75 .

\section{APPENDIX}

Appendix 7.1. Proof of Lemma 1. To simplify the formulas, let us denote

$$
\begin{aligned}
\mathcal{A}_{\text {xoy }} & =\left|\begin{array}{lll}
x_{1} & y_{1} & 1 \\
x_{2} & y_{2} & 1 \\
x_{3} & y_{3} & 1
\end{array}\right|, \quad \mathcal{A}_{y o z}=\left|\begin{array}{lll}
y_{1} & z_{1} & 1 \\
y_{2} & z_{2} & 1 \\
y_{3} & z_{3} & 1
\end{array}\right|, \quad \mathcal{A}_{z o x}=\left|\begin{array}{lll}
z_{1} & x_{1} & 1 \\
z_{2} & x_{2} & 1 \\
z_{3} & x_{3} & 1
\end{array}\right|, \\
d_{T} & =\left|\begin{array}{lll}
x_{1} & y_{1} & z_{1} \\
x_{2} & y_{2} & z_{2} \\
x_{3} & y_{3} & z_{3}
\end{array}\right|, \quad \mathcal{A}=\sqrt{\mathcal{A}_{x o y}^{2}+\mathcal{A}_{y o z}^{2}+\mathcal{A}_{z o x}^{2}} .
\end{aligned}
$$

Then the equation of the plane $M_{1} M_{2} M_{3}$ is

$$
\mathcal{A}_{\text {yoz }} x+\mathcal{A}_{z o x} y+\mathcal{A}_{\text {xoy }} z-d_{T}=0
$$

and if we project this plane onto $x O y$ in order to transform the integral on $\Omega$ to a double integral, we obtain

$$
d \Omega(\mathbf{x})=d \Omega(x, y, z)=\frac{|\mathcal{A}|}{\left|\mathcal{A}_{x o y}\right|} d x d y .
$$

Note that if the triangle $T$ is parallel to $O z$, then $\mathcal{A}_{x o y}=0$, and in this case we prove the formula by projecting the triangle $\left[M_{1} M_{2} M_{3}\right]$ onto the plane $x O z$. So,

$$
\begin{aligned}
\phi_{1}(x, y, z(x, y)) & =a x+b y-c \cdot \frac{x \mathcal{A}_{y o z}+y \mathcal{A}_{z o x}-d_{T}}{\mathcal{A}_{x o y}} \\
& =a x+b y-\frac{c}{\mathcal{A}_{x o y}} \cdot\left|\begin{array}{cccc}
x & y & 0 & 1 \\
x_{1} & y_{1} & z_{1} & 1 \\
x_{2} & y_{2} & z_{2} & 1 \\
x_{3} & y_{3} & z_{3} & 1
\end{array}\right|=A x+B y+C .
\end{aligned}
$$


Then

$$
\begin{aligned}
f_{1} & =\phi_{1}\left(x_{1}, y_{1}, z\left(x_{1}, y_{1}\right)\right)=a x_{1}+b y_{1}-\frac{c}{\mathcal{A}_{x o y}} \cdot\left|\begin{array}{cccc}
x_{1} & y_{1} & 0 & 1 \\
x_{1} & y_{1} & z_{1} & 1 \\
x_{2} & y_{2} & z_{2} & 1 \\
x_{3} & y_{3} & z_{3} & 1
\end{array}\right| \\
& =a x_{1}+b y_{1}-\frac{c}{\mathcal{A}_{x o y}}\left(-z_{1}\right) \mathcal{A}_{x o y}=a x_{1}+b y_{1}+c z_{1},
\end{aligned}
$$

and analogously for the values $f_{2}, f_{3}, g_{1}, g_{2}, g_{3}$.

Coming back to the integral and using the formula for the integral of a Bernsteintype polynomial (see 4 , $\S 5$ ), we may write

$$
\begin{aligned}
\int_{T} \phi_{1}(\mathbf{x}) \phi_{2}(\mathbf{x}) d \Omega(\mathbf{x}) \\
\quad=\int_{\operatorname{Pr}_{x o y} T} \frac{2 a(T)}{\left|\mathcal{A}_{x o y}\right|} \phi_{1}(x, y, z(x, y)) \phi_{2}(x, y, z(x, y)) d x d y \\
=\frac{2 a(T)}{\left|\mathcal{A}_{x o y}\right|} \cdot \frac{a\left(\operatorname{Pr}_{x o y} T\right)}{12}\left(f_{1} g_{1}+f_{2} g_{2}+f_{3} g_{3}+\left(f_{1}+f_{2}+f_{3}\right)\left(g_{1}+g_{2}+g_{3}\right)\right) \\
=\frac{a(T)}{12}\left(f_{1} g_{1}+f_{2} g_{2}+f_{3} g_{3}+\left(f_{1}+f_{2}+f_{3}\right)\left(g_{1}+g_{2}+g_{3}\right)\right)
\end{aligned}
$$

since $\left|\mathcal{A}_{\text {xoy }}\right|=2 a\left(\operatorname{Pr}_{\text {xoy }} T\right)$, as $\operatorname{Pr}_{\text {xoy }} T$ denotes the projection of the triangle $T$ onto the plane $x O y$.

Appendix 7.2. Proof of Proposition 4, We focus on the face $\Delta$ of the polyhedron $\Pi$, contained in a plane $\mathcal{P}$ of equation $a x+b y+c z+d=0$. At least one of the numbers $a, b, c$ is nonzero. Without loss of generality, we suppose $c \neq 0$ and consider the parametric equations of the plane $\mathcal{P}$

$$
x(u, v)=u, y(u, v)=v, z(u, v)=-\frac{1}{c}(a u+b v+d), \quad(u, v) \in \mathbb{R}^{2} .
$$

We intend to express $d \omega(\boldsymbol{\eta})$ and $d \Omega(\mathbf{x})$ with respect to $d u$ and $d v$.

With $\vec{r}(u, v)=\left(u, v,-\frac{1}{c}(a u+b v+d)\right)$ representing the vectorial equation of the plane $\mathcal{P}$ and $\vec{r}_{u}=\left(1,0,-\frac{a}{c}\right), \vec{r}_{v}=\left(0,1,-\frac{b}{c}\right)$ its partial derivatives, we have $\vec{r}_{u} \times \vec{r}_{v}=\left(\frac{a}{c}, \frac{b}{c}, 1\right)$ and therefore

$$
d \Omega(\mathbf{x})=\left\|\vec{r}_{u} \times \vec{r}_{v}\right\| d u d v=\frac{\sqrt{a^{2}+b^{2}+c^{2}}}{|c|} d u d v .
$$

The projection onto the sphere of the point $(x, y, z) \in \Delta$ has the coordinates

$$
\begin{aligned}
& X(x, y, z)=\frac{x}{\sqrt{x^{2}+y^{2}+z^{2}}}, \\
& Y(x, y, z)=\frac{y}{\sqrt{x^{2}+y^{2}+z^{2}}}, \\
& Z(x, y, z)=\frac{z}{\sqrt{x^{2}+y^{2}+z^{2}}} .
\end{aligned}
$$


If we want to express $d \omega(\boldsymbol{\eta})$ with respect to $d u$ and $d v$, we have to consider $x=$ $x(u, v), y=y(u, v), z=z(u, v)$ given by (18). Denoting

$$
\begin{aligned}
\vec{R}(u, v)= & (X(x(u, v), y(u, v), z(u, v)), \\
& Y(x(u, v), y(u, v), z(u, v)), \\
& Z(x(u, v), y(u, v), z(u, v))),
\end{aligned}
$$

we have

$$
\begin{aligned}
\vec{R}_{u} & =\left(X_{u}, Y_{u}, Z_{u}\right) \\
& =\left(X_{x} x_{u}+X_{y} y_{u}+X_{z} z_{u}, Y_{x} x_{u}+Y_{y} y_{u}+Y_{z} z_{u}, Z_{x} x_{u}+Z_{y} y_{u}+Z_{z} z_{u}\right) \\
& =\left(\nabla X \cdot \vec{r}_{u}, \nabla Y \cdot \vec{r}_{u}, \nabla Z \cdot \vec{r}_{u}\right)
\end{aligned}
$$

and similarly

$$
\vec{R}_{v}=\left(\nabla X \cdot \vec{r}_{v}, \nabla Y \cdot \vec{r}_{v}, \nabla Z \cdot \vec{r}_{v}\right) .
$$

Therefore, using the formula

$$
\left(\vec{u}_{1} \cdot \vec{v}_{1}\right)\left(\vec{u}_{2} \cdot \vec{v}_{2}\right)-\left(\vec{u}_{1} \cdot \vec{v}_{2}\right)\left(\vec{u}_{2} \cdot \vec{v}_{1}\right)=\left(\vec{u}_{1} \times \vec{u}_{2}\right)\left(\vec{v}_{1} \times \vec{v}_{2}\right),
$$

we further obtain

$$
\vec{R}_{u} \times \vec{R}_{v}=(\vec{M} \cdot \vec{n}, \vec{N} \cdot \vec{n}, \vec{P} \cdot \vec{n})
$$

with

$$
\vec{M}=\nabla Y \times \nabla Z, \quad \vec{N}=\nabla Z \times \nabla X, \quad \vec{P}=\nabla X \times \nabla Y, \quad \vec{n}=\vec{r}_{u} \times \vec{r}_{v},
$$

and consequently

$$
\left\|\vec{R}_{u} \times \vec{R}_{v}\right\|^{2}=(\vec{M} \cdot \vec{n})^{2}+(\vec{N} \cdot \vec{n})^{2}+(\vec{P} \cdot \vec{n})^{2} .
$$

Evaluating $\vec{M} \cdot \vec{n}$, we get

$$
\vec{M} \cdot \vec{n}=\frac{1}{c}\left|\begin{array}{ccc}
a & b & c \\
Y_{x} & Y_{y} & Y_{z} \\
Z_{x} & Z_{y} & Z_{z}
\end{array}\right|=\frac{x}{c\left(x^{2}+y^{2}+z^{2}\right)^{2}}(a x+b y+c z)=-\frac{x d}{c\left(x^{2}+y^{2}+z^{2}\right)^{2}}
$$

and analogous expressions for $\vec{N} \cdot \vec{n}$ and $\vec{P} \cdot \vec{n}$. Finally

$$
d \omega(\boldsymbol{\eta})=\left\|\vec{R}_{u} \times \vec{R}_{v}\right\| d u d v=\frac{|d|}{|c|\left(x^{2}+y^{2}+z^{2}\right)^{3 / 2}} d u d v .
$$

Comparing (19) and (20), we obtain

$$
d \omega(\boldsymbol{\eta})=\frac{|d|}{\sqrt{a^{2}+b^{2}+c^{2}}} \cdot \frac{1}{\left(x^{2}+y^{2}+z^{2}\right)^{3 / 2}} d \Omega(\mathbf{x})
$$

and thus formula (5) is proved.

Furthermore, using formula (3), we express $x, y$, and $z$ with respect to $\boldsymbol{\eta}=$ $\left(\eta_{1}, \eta_{2}, \eta_{3}\right)$ and we get

$$
\left(x^{2}+y^{2}+z^{2}\right)^{3 / 2}=\left(\frac{d^{2}\left(\eta_{1}^{2}+\eta_{2}^{2}+\eta_{3}^{2}\right)}{\left(a \eta_{1}+b \eta_{2}+c \eta_{3}\right)^{2}}\right)^{3 / 2}=\frac{d^{3}}{\left(a \eta_{1}+b \eta_{2}+c \eta_{3}\right)^{3}} .
$$

Replacing it in (5), we obtain formula (6). 


\section{ACKNOWLEDGMENTS}

The author thanks Jürgen Prestin for many productive discussions and the referees for their helpful suggestions in improving the presentation and organization of this material.

\section{REFERENCES}

1. G. P. Bonneau, Optimal Triangular Haar Bases for Spherical Data, in IEEE Visualization '99, San Francisco, CA, 1999.

2. A. Cohen, L. M. Echeverry and Q. Sun, Finite Elements Wavelets, Report, University Pierre et Marie Curie, Paris, 2000.

3. S. Dahlke, W. Dahmen and I. Weinreich, Multiresolution Analysis and Wavelets on $\mathbb{S}^{2}$ and $\mathbb{S}^{3}$, Numer. Funct. Anal. Optim. 16 (1995), 19-41. MR1322896 (96a:42044)

4. M. Floater and E. Quak, Piecewise Linear Prewavelets on Arbitrary Triangulations, Numer. Math. 82 (1999), 221-252. MR.1685460 (2000a:42053)

5. M. Floater and E. Quak, A Semi-prewavelet Approach to Piecewise Linear Prewavelets on Triangulation, Approximation Theory IX (C.K. Chui and L.L. Schumaker, eds.), vol. 2, Vanderbilt University Press, 1998, pp. 63-70. MR1743034

6. M. Floater and E. Quak, Linear Independence and Stability of Piecewise Linear Prewavelets on Arbitrary Triangulations, SIAM J. Numer. Anal. 38 2000, no. 1, 58-79. MR1770342 (2001e:65237)

7. M. Floater, E. Quak and M. Reimers, Filter Bank Algorithms for Piecewise Linear Prewavelets on Arbitrary Triangulation, J. Comp. Appl. Math. 119 (2000), 185-207. MR.1774217 (2001i:65145)

8. W. Freeden and U. Windheuser, Combined Spherical Harmonics and Wavelet Expansions A Future Concept in Earth's Gravitational Potential Determination, Appl. Comput. Harm. Anal. 4 (1997), 1-37. MR.1429676 (97j:86004)

9. J. Göttelmann, Locally Supported Wavelets on Manifolds with Applications to the 2D Sphere, Appl. Comput. Harm. Anal. 7 (1999), 1-33. MR1699606 (2000j:42051)

10. M. Lounsbery, T. DeRose and J. Warren, Multiresolution Analysis for Surfaces of Arbitrary Topological Type, ACM Transactions on Graphics 16 (1997), no. 1, 34-73.

11. F. Narcowich and J. D. Ward, Wavelets Associated with Periodic Basis Functions, Appl. Comput. Harm. Anal. 3 (1996), 40-56. MR1374394 (96j:42022)

12. F. Narcowich and J. D. Ward, Nonstationary Wavelets on the $m$-Sphere for scattered Data, Appl. Comput. Harm. Anal. 3 (1996), 324-336. MR1420501 (97h:42020)

13. G. Nielson, I. Jung and J. Sung, Haar Wavelets over Triangular Domains with Applications to Multiresolution Models for Flow over a Sphere, IEEE Visualization '97, IEEE 1997, pp. 143-150.

14. P. Oswald, Multilevel Finite Element Approximation: Theory and Applications, B. G. Teubner, Stuttgart, 1994. MR.1312165 (95k:65110)

15. D. Potts and M. Tasche, Interpolatory Wavelets on the Sphere, Approximation Theory VIII (C. K. Chui and L. L. Schumaker, eds.), vol. 2, World Scientific, Singapore, 1995, pp. 335-342. MR $1471800(98 \mathrm{e}: 42040)$

16. D. Potts, G. Steidl and M. Tasche, Kernels of Spherical Harmonics and Spherical Frames, Advanced Topics in Multivariate Approximations (F. Fontanella, K. Jetter, P-J. Laurent, eds.), World Scientific, Singapore, 1996. MR.1661417 (99g:42042)

17. D. Roşca, Haar wavelets on spherical triangulations, in Advances in Multiresolution for Geometric Modelling (N. A. Dodgson, M. S. Floater, M. A. Sabin, eds.), Springer Verlag, 2005, pp. 405-417.

18. D. Roşca, Optimal Haar wavelets on spherical triangulations, Pure Mathematics and Applications, Budapest (to appear).

19. D. Roşca, Piecewise Constant Wavelets Defined on Closed Surfaces, J. Comput. Anal. Appl. (to appear).

20. H. Schaeben, D. Potts and J. Prestin, Spherical Wavelets with Application in Preferred Crystallographic Orientation, IAMG' 2001, Cancun, 2001.

21. P. Schröder and W. Sweldens, Spherical Wavelets: Efficiently Representing Functions on the Sphere, Computer Graphics Proceedings (SIGGRAPH 95), 1995, pp. 161-172. 
22. P. Schröder and W. Sweldens, Spherical Wavelets: Texture Processing, preprint.

23. L. Schumaker and C. Traas, Fitting Scattered Data on Sphere-like Surface Using Tensor Products of Trigonometric and Polynomial Splines, Numer. Math. 60 (1991), 133-144. MR.1131503 (92j:65012)

24. I. Weinreich, A Construction of $C^{1}$-Wavelets on the Two-Dimensional Sphere, Appl. Comput. Harm. Anal. 10 (2001), 1-26. MR1808197 (2001k:42048)

Institute of Mathematics, University of Lübeck, Wallstrasse 40, Lübeck 23560, GerMANY

Current address: Department of Mathematics, Technical University of Cluj-Napoca, str. Daicoviciu 15, Cluj-Napoca 400020, Romania

E-mail address: rosca@math.uni-luebeck.de, Daniela.Rosca@math.utcluj.ro 